\title{
Educación superior y crecimiento económico en Colombia (1971-2016): una relación de cointegración.
}

\author{
Brayan Alexander Baron Ortegon.
}

babarono@unal.edu.co

\begin{abstract}
This article analyzes the relation between GDP per capita (CPIBpc) and access to tertiary education, seen from the perspective of growth rate of the number of enrollments (TCMes) in higher education in Colombia for the period (1971-2016). By using a VEC model and assuming everything else constant, it is concluded that TCMes Granger caused the Colombian GDP per capita and vice-versa, therefore, the existence of a long run relation between both variables is verified. This result helps to explain the dynamics of Colombian economic growth per capita of the last forty-five years and the impact of the accumulation of human capital on it.
\end{abstract}

\section{Keywords}

Economic growth; Human Capital; Access to higher education; Colombia; VEC model.

JEL codes: 040, 047, I26, I25.

\section{Resumen}

En este artículo se analiza la relación entre crecimiento económico per cápita(CPIBpc) y la cobertura educativa terciaria, vista desde la tasa de crecimiento del número de matrículas (TCMes) en educación superior en Colombia para el periodo de estudio (1971-2016), usando un modelo VEC. Suponiendo todo lo demás constante, se concluye que TCMes causo en el sentido de Granger al crecimiento económico colombiano per cápita y viceversa, por lo tanto, se comprueba la existencia de una relación de equilibrio en el largo plazo entre estas dos variables. Este resultado contribuye a explicar la dinámica del crecimiento económico colombiano per cápita de los últimos cuarenta y cinco años y el impacto de la acumulación de capital humano sobre este.

\section{Palabras claves}

Crecimiento económico; Cobertura educativa; Capital humano; Colombia; Educación superior, Modelo VEC.

Códigos JEL: O40, 047, I26, I25. 


\section{Marco teórico}

Usamos el Modelo de Solow ampliado ${ }^{1}$, para incluir al capital humano, tal como se presenta inicialmente en Romer (2012), aunque en este artículo, le damos un tratamiento ligeramente diferente. Se parte de una función de producción agregada típica Cobb-Douglas (Ecuación 2), con tres factores de producción: K, capital físico, el cual se deprecia a una tasa constante $\delta ; \mathrm{A}$, tecnología, bien no rival y replicable que crece a una tasa exógena g: $\dot{A}_{t}=g A_{T} ; \mathrm{H}$ que representa la cantidad de servicios productivos por trabajador: $H_{t}=L_{t} G(E)_{t}$ donde:

Donde, L representa la cantidad de trabajadores y G(E) representa el capital humano por trabajador en función del número de años de educación por trabajador. Como de costumbre, el número de trabajadores crece a una tasa exógena n: $\dot{L}_{t}=n L_{T}$; (Romer, 2002, p.137).

Por lo que la función de producción agregada es:

$$
\left.Y=K^{\alpha}[A L H]^{1-\alpha}, \text { donde } 0<\alpha<1 \text { (Ecuación } 1\right) \text {. }
$$

El aporte total de servicios productivos del trabajo es:

$$
H_{t}=L_{t} G(E)_{t} \text { (Ecuación 2). }
$$

$(E)_{t}$ representa los años de estudio promedio en la economía, en el año t. Romer (2002) afirma que los resultados microeconómicos llevan a pensar que $\mathrm{G}^{\prime}(\mathrm{E})>0$ y $\mathrm{G}^{\prime \prime}(\mathrm{E})>0$ y dice que una función que cumple con estas dos características es:

$$
G(E)=e^{\phi E},(\phi>0) \wedge(E \geq 0) \text { (Ecuación 3). }
$$

Si reemplazamos las ecuaciones 2 y 3, en la ecuación 1, tenemos que la función de producción es:

$$
\mathrm{Y}=K^{\alpha} A^{1-\alpha} L^{1-\alpha} e^{\phi(E)(1-\alpha)}(\text { Ecuacion } 3), \text { donde }(\phi>0) \wedge(E \geq 0)
$$

Romer (2002), supone que la dotación total de servicios del trabajo es igual a: LG(E), que a su vez es la suma del trabajo primario: LG (0) y el capital humano: LG(E), $E \geq 0$. En este trabajo

\footnotetext{
${ }^{1}$ Este modelo está parcialmente basado en el presentado por David Romer en Romer (2002): Macroeconomía Avanzada.
} 
supondremos que el trabajo primario es igual a $L G(0 \leq E \leq 13$.), es decir consideramos como trabajo primario, aquellas contribuciones hechas por personas que no han cursado al menos un año de educación superior. Por lo que el capital humano en nuestro caso es: $\mathrm{H}=\mathrm{LG}$ ( $E=S \geq$ 13.), solo aquella parte de los servicios prestados por los trabajadores con al menos un año de educación superior, es decir grado de nivel técnico, tecnológico, licenciatura, pregrado, especialización, maestría o doctorado. Donde $S_{t}$, representa los años de educación superior promedio en el año t. Suponemos que nadie repite un año escolar, por lo que, al momento de graduarse de la primaria, debió haber acumulado solo 6 años de estudio y del bachillerato solo 12. Por lo tanto, teniendo en cuenta estos supuestos la función de producción agregada es:

$$
Y=f(\mathrm{~K}, \mathrm{~A}, \mathrm{~L})=A K^{\alpha}[L G(S)]^{1-\alpha} \text {, donde } 0<\alpha<1 \text { y } 13 \leq S<T \text { (Ecuación 4). }
$$

Tomando la derivada parcial de Y respecto a S:

$$
\begin{aligned}
& \frac{\partial Y}{\partial S}=K^{\alpha} A^{1-\alpha} L^{1-\alpha} e^{\phi(E)(1-\alpha)}>0 ; \text { por lo cual la función es creciente. } \\
& \frac{\partial^{2} Y}{(\partial S)^{2}}=K^{\alpha} A^{1-\alpha} L^{1-\alpha} e^{\phi(E)(1-\alpha)}>0 ; \text { por lo cual la función es convexa. }
\end{aligned}
$$

Definiendo a $y=\frac{Y}{A L G(S)^{\prime}}$, como el ingreso por unidad de servicios efectivos de trabajo y a $r=$ $\frac{K}{A L G(S)}$, como el capital físico por unidad de servicios efectivos de trabajo (Romer, 2012), que en nuestro caso serian solo aquellos aportados por el capital humano. Tenemos que:

$$
\dot{r}=s r_{t}^{\alpha}-(\mathrm{n}+\mathrm{g}+\delta) r(\text { Ecuación 5). }
$$

Dada una acumulación de capital creciente y una tasa de ahorro constante; s, cuando se alcance un nivel de capital por servicios efectivos de trabajo $r^{*}=\left(\frac{s}{n+g+\delta}\right)^{1-\alpha}$, la tasa de crecimiento del capital por unidad de servicios efectivos del trabajo será cero: $\dot{r}=0$ y la economía habrá alcanzado el estado estacionario (Romer, 2002). Desde ese punto, la senda de crecimiento sostenido del ingreso depende de la tasa de crecimiento de la tecnología: g, la cual es exógena al modelo.

Dado que, en lo anterior, se ha trabajado con $L_{t}$, que representa el número de trabajadores y con el ingreso por unidad de servicios efectivos de trabajo, pero como lo que interesa es la población en conjunto y por lo tanto el ingreso per capita. Romer (2012), supone una tasa de natalidad, $\mathrm{n}$, un número de años de vida $\tau$; al número de personas que nacen en t como $B_{t}$, con lo anterior $N_{t}$ que es el número de personas vivas en t es igual a: 


$$
\begin{gathered}
N_{t}=\int_{\tau=0}^{\tau} B(t-\tau) d \tau=\int_{\tau=0}^{\tau} B_{t} e^{-n \tau} d \tau=\frac{1-e^{-n \tau}}{n} B_{t} \\
L_{t}=\int_{\tau=S}^{\tau} B(t-\tau) d \tau=\int_{\tau=S}^{\tau} B_{t} e^{-n \tau} d \tau=\frac{e^{-n S}-e^{-n \tau}}{n} B_{t} \\
\frac{L_{t}}{N_{t}}=\frac{e^{-n S}-e^{-n \tau}}{1-e^{-n \tau}} \text { (Ecuación 6.) (Romer, 2012). }
\end{gathered}
$$

Usando la ecuación 6, hallamos el ingreso per-capital del estado estacionario, donde la senda de crecimiento es jalonada por la tasa de crecimiento de la tecnología, g:

$$
\left(\frac{Y_{t}}{N_{t}}\right)^{*}=y^{*} A_{t} G(S) \frac{e^{-n S_{-}}-n \tau}{1-e^{-n \tau}} \quad \text { (Ecuación 7.) (Romer, 2012). }
$$

En la ecuación 7, ni A ni y dependen de S, pues por supuesto A depende de una tasa de crecimiento exógena; e y depende de $r^{*}=\left(\frac{s}{n+g+\delta}\right)^{1-\alpha}$. Asi que, un cambio en $\mathrm{S}$ en la ecuación 7 , afecta única y directamente al ingreso per capita del estado estacionario; $\left(\frac{Y_{t}}{N_{t}}\right)^{*}$ (Romer, 2012). El efecto de un aumento de $\mathrm{S}$ sobre el ingreso per capita es ambiguo, pues al aumentar $\mathrm{G}(\mathrm{s})$, aumenta el acervo de capital humano y así el ingreso per capita, pero al mismo tiempo $\left(\frac{e^{-n S}-e^{-n \tau}}{1-e^{-n \tau}}\right.$ ) que es la proporción de gente que trabaja $\frac{L_{t}}{N_{t}}$, disminuye por lo que el ingreso per capita disminuye ${ }^{2}$ (Romer, 2002) ¿Cuál efecto domina al otro?_Depende del horizonte temporal, en el corto plazo el aumento de $S$ lleva a una disminución en el ingreso per capita, (12 -S), luego de lo cual $(s-\tau)$, el ingreso per capita aumenta, debido a la incorporación al mercado laboral de la población de nuevos trabajadores y su capital humano, que aumentan el nivel promedio de educación superior en G(S). El nivel óptimo de S que maximiza el ingreso per capita en la senda de crecimiento de largo plazo, es decir luego de haber alcanzado el estado estacionario es:

$$
(S)^{*}=\tau-\frac{1}{n} \ln \left(\frac{\phi}{\phi-n}\right) \text { (Ecuación 8.) (Romer, 2012). }
$$

2 Esto es especialmente cierto en aquellas personas que cursan sus licenciaturas, pregrados o doctorados, pues son actividades que la mayoría de las veces requieren dedicación exclusiva. Por lo que, en este artículo, suponemos que nadie trabaja ni estudia al mismo tiempo, lo que a primera vista parece un supuesto razonable. 
$\frac{\delta S}{\delta \tau}=1$, por lo que un aumento en la esperanza de vida aumenta en uno la cantidad de educación superior, S, óptima para maximizar el ingreso per capita en el largo plazo. $\frac{\delta S}{\delta n}<$ 0 (Romer, 2012), por lo que una disminución de la tasa de natalidad aumenta la cantidad de educación superior, S, óptima para maximizar el ingreso per capita en el largo plazo. Esto va en línea con Barro (2013), que, en un estudio para aproximadamente 100 países, desde 1960 a 1990, encontró que la tasa de natalidad y el crecimiento económico per capita estuvieron correlacionados negativamente.

Existe un problema de medir el capital humano solo a través de los años de escolaridad o calidad educativa y es que la educación no es la única fuente de capital humano, otros aspectos como la salud, nutrición en la niñez y adolescencia, impactan también en el desempeño futuro de los trabajadores (Hanuseck, 2013).

La mejora del capital humano individual es fuente de ganancias potenciales para los individuos, pues esta correlacionado con mejoras en la productividad y por lo tanto en los salarios y en las perspectivas de empleo, al mismo tiempo que reduce el riesgo de desempleo (Mercan y Sezer, 2014).

la aproximación neoclásica del modelo de Solow con capital humano incluido ha sido usualmente usada para explicar las diferencias entre ingreso per capita de diferentes países y el proceso de convergencia en el largo plazo hacia un nivel de equilibrio más alto (Gumus (2005 citado por).

El modelo de crecimiento de Solow con capital humano ha mostrado resultados consistentes en el largo plazo, de hecho esa fue una de las razones para haberlo seleccionado como referente teórico en este artículo, aunque según algunos autores no diferencia exitosamente entre capital humano y capital físico (Dahli (2002) citado por Mercan y Sezer (2014)). Así que debemos ser precavidos con las conclusiones aquí hechas, pues las definiciones de capital e ingreso por servicios por unidad de servicios efectivos de trabajo son poco intuitivas, debido a que en el denominador de sus expresiones, se conjugan elementos que a primera vista, parecen muy dispares como tecnología, trabajo humano y años de educación. A pesar de ello, creemos que es una abstracción útil, en cuanto permite aislar sus efectos individualmente para hacernos una idea de la evolución, al menos en teoría, en el largo plazo del ingreso per capita.

Como es de esperar, un aumento en el capital humano tiene efectos relativamente mayores en aquellos sectores donde es usado más intensivamente (Hirsch y Sulls (2009) citados por Mercan y Sezer (2014)) Por esta razón, los países desarrollados tienen una ventaja sobre los países en desarrollo, ya que los primeros son capaces de cerrar rápidamente la brecha entre los procesos innovadores y de alta tecnología y el sistema educativo, se internalizan más rápidamente (Mercan y Sezer, 2014). Ello no sucede generalmente en los países en desarrollo, quienes son en general dependientes de la transferencia de tecnología y donde los procesos de innovación son menos dinámicos. En los países en desarrollo, un mejor nivel educativo promedio, está más relacionado no con la producción de nuevas tecnologías y conocimientos, sino con una mayor capacidad de absorción y transferencia de tecnología y conocimientos desde los países desarrollados (Barro, 2013). 


\section{Análisis econométrico}

\subsection{Modelo VEC}

Para estudiar la relación entre el stock de capital humano, visto desde la cobertura en educación terciaria o superior y el crecimiento económico en Colombia, es usado un modelo VEC (Vector de corrección de errores). El cual, bajo el cumplimiento de ciertas condiciones previas, permite estudiar la relación de equilibrio en largo plazo entre variables y que tan rápido regresa a él, luego de choques en el corto plazo (Novales, 2014).

Según EViews (2018), para el caso de un sistema de ecuaciones con dos series de tiempo, una sola ecuación de cointegración entre ellas $\left(y_{2 t}=\aleph_{1} y_{1 t}+c_{1}\right)$ y un rezago de una sola diferencia. El modelo VEC se puede represntar como:

$\Delta y_{1, t}=\alpha\left(y_{1, t-1}-\aleph_{11} y_{2, t-1}-c_{1}\right)+\aleph_{12} \Delta y_{1, t-1}+\aleph_{13} \Delta y_{2 t-1}+K_{1}+\varepsilon_{1 t}$ (Ecuación 9)

Donde, $\Delta y_{1, t-} 1$ t es la primera diferencia de la primera variable endógena; $y_{1, t-1}$ es la primera variable endógena en el period anterior; $\aleph_{11}$ es el coeficiente de la ecuacion de cointehracin; $c_{1}$ es el termino constant de la ecuacion de cointegracion; $\aleph_{12}$ es el coeficiente del término error de la primera variable rezagada una vez $; \aleph_{13}$ es el coeficiente del termino error de la segunda variable con un rezago; $\varepsilon_{1 t}$ son lor errores de la primera ecuación del modelo biecuacional VEC; $\mathrm{K}_{1}$ es el termino constante de la primera ecuación del sistema; $\alpha$ es el coeficiente del término de corrección de errores de la primera ecuación, debe ser estrictamente menor a cero: $\alpha<0$, mide la velocidad de ajuste de la primera variable hacia el equilibrio de largo plazo (EViews, 2018). La segunda ecuación es análoga a la primera. Para este caso, ambas series de tiempo deben cumplir con las siguientes condiciones:

1. $y_{1 t}$ e $y_{2 t}$ están cointegradas y su orden de cointegración es de orden 1: I (1):

2. $\varepsilon_{1 t} \mathrm{y} \quad \varepsilon_{2 t}$ son ruido blanco, sus esperanzas son constantes para cualquier subintervalo de tiempo, $: \mathrm{E}\left[\varepsilon_{1 t}\right]=c$ and $\mathrm{E}\left[\varepsilon_{2 t}\right]=c$; al igual que sus varianzas: $\sigma_{\varepsilon_{1 t}}^{2}=\mathrm{c}$ and $\sigma_{\varepsilon_{1 t}}^{2}=\mathrm{c}$, where c $\in R^{+}$, including zero.

3. $\operatorname{Cov}\left(\varepsilon_{1 t}, \varepsilon_{2 t}\right)=\sigma_{\varepsilon_{1 t} \varepsilon_{2 t}}$ 


\subsection{Análisis grafico}
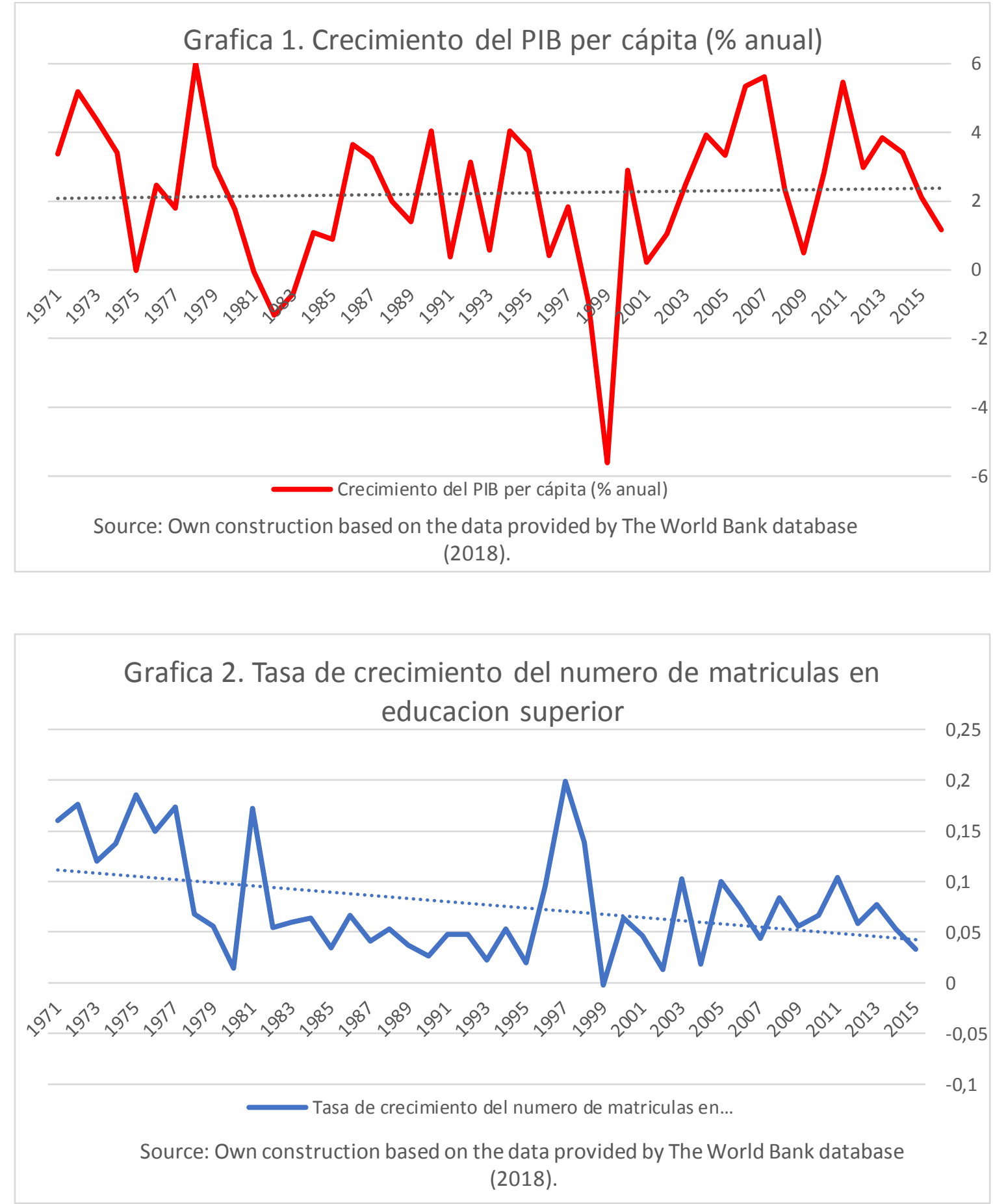

En el periodo 1971-2016, el crecimiento promedio del ingreso per capita fue del 2,2\% , a su vez el crecimiento promedio de la tasa de crecimiento del número de matrículas en educación superior fue del $7.63 \%$, el cual fue mucho mayor al crecimiento poblacional promedio, el cual 
fue del 1.71\% . Si observamos, la elasticidad número de matrículas en educación superior del ingreso per capita: $\varepsilon_{Y p c}$; esta es igual a 1.04, es decir es elástica (Banco Mundial, 2018).

Al observar las Gráficas 1 y 2, ambas series muestran tendencias crecientes. Estas podrían ser deterministas o estocásticas. Al modelar cada una de las series contra el tiempo, para observar si el coeficiente de la variable tiempo era significativa o no y por lo tanto si su tendencia era determinista (función del tiempo) o estocástica. Se encontró que para la serie de Crecimiento del PIB per cápita, dicho coeficiente era de 0.86 , por lo que no es significativo y asumimos que la serie tiene una tendencia estocástica (Véase Tabla 1). Al realizar el mismo proceso para la serie de Tasa de crecimiento del número de matrículas en educación superior ${ }^{3}$, se halló que el coeficiente de la variable exógena tiempo, si era significativo, por lo que asumimos que dicha serie, tiene una tendencia determinística (Véase Tabla 2):

\section{Tabla 1.}

\begin{tabular}{|c|c|c|c|c|}
\hline \multicolumn{5}{|c|}{$\begin{array}{l}\text { Dependent Variable: CRECIMIENTO_DEL_P } \\
\quad \text { AL } \\
\text { Method: Least Squares } \\
\text { Date: } 08 / 15 / 18 \text { Time: } 11: 35 \\
\text { Sample (adjusted): } 247 \\
\text { Included observations: } 46 \text { after adjustments }\end{array}$} \\
\hline Variable & Coefficient & Std. Error & $\mathrm{t}$-Statistic & Prob. \\
\hline TIEMPO & 0.00 & 0.024405 & 0.1 & 0.8615 \\
\hline C & 2.190318 & 0.680050 & 3.220817 & 0.0024 \\
\hline R-squared & 0.000699 & \multicolumn{2}{|c|}{ Mean dependent var } & 2.295228 \\
\hline Adjusted R-squared & -0.022012 & \multicolumn{2}{|c|}{ S.D. dependent var } & 2.173631 \\
\hline S.E. of regression & 2.197424 & \multicolumn{2}{|c|}{ Akaike info criterion } & 4.45495 \\
\hline Sum squared resid & 212.4615 & \multicolumn{2}{|c|}{ Schwarz criterion } & 4.53445 \\
\hline Log likelihood & -100.4639 & \multirow{2}{*}{\multicolumn{2}{|c|}{$\begin{array}{l}\text { Hannan-Quinn criter. } \\
\text { Durbin-Watson stat }\end{array}$}} & 4.484736 \\
\hline F-statistic & 0.030787 & & & 1.327298 \\
\hline Prob(F-statistic) & 0.861522 & & & \\
\hline
\end{tabular}

\section{Fuente: Banco Mundial (2018). Estimación realizada en EViews.}

\section{Tabla 2 .}

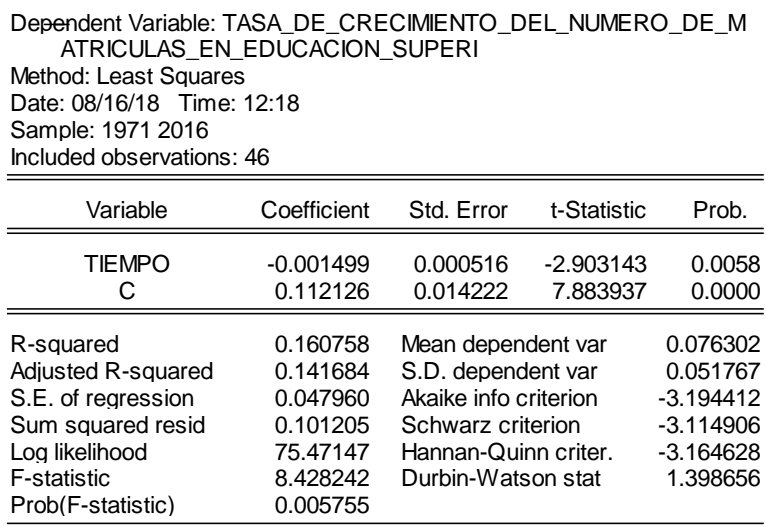

\section{Fuente: Banco Mundial (2018). Estimación realizada en EViews.}

\footnotetext{
${ }^{3}$ Dado que el valor de la serie del número de matrículas en educación superior, para el año 2003 no estaba disponible, se tomó como su proxy, el valor promedio de los tres años anteriores : 2000, 2001 y 2002 y el de los tres años posteriores 2004, 2005 y 2006. Con dicho valor, se construyó la serie de tasa de crecimiento del número de matrículas en educación superior, para el periodo 1971-2016.
} 


\subsection{Prueba de raíz unitaria}

Según Hamilton (1994), la prueba de Dickey-Fuller aumentada (ADF)permite contrastar la hipótesis nula de que la serie tiene una raíz unitaria y por lo tanto no es estacionaria, con la hipótesis alternativa de que es estacionaria o no tiene raíz unitaria. Al realizar la prueba, especificándola para un número máximo de 5 rezagos y a un nivel de significancia de: $\alpha=0.05$, se encontró que ninguna de las dos series bajo estudio era estacionarias.

En la Grafica 1, la serie del crecimiento del PIB per cápita muestra una leve tendencia creciente, por lo tanto, realizamos la prueba $\mathrm{ADF}$, incluyendo una tendencia y un intercepto. Especificando el test para un máximo de 5 rezagos, se obtiene un valor p de 0.44 , por lo tanto, existe evidencia estadística para aceptar la hipótesis nula y considerar la serie del crecimiento del PIB per cápita como no estacionaria (Véase Anexo 1).

En la gráfica 2, la serie de la tasa de crecimiento del número de matrículas en educación superior muestra una clara tendencia decreciente, por lo tanto, realizamos la prueba ADF, especificando el test para un máximo de 5 rezagos, incluyendo una tendencia y un intercepto. Se obtiene un valor p de 0.24 , por lo tanto, existe evidencia estadística para aceptar la hipótesis nula y considerar la Tasa de crecimiento del número de matrículas en educación superior como no estacionaria (Véase Anexo 2).

\subsection{Orden de integración}

Una serie no estacionaria $\left(y_{i t}\right)$ tiene un orden de integración d: I (d), si es necesario diferenciarla $\mathrm{d}$ veces para que la nueva serie diferenciada sea estacionaria. En nuestro caso, al diferenciar cada una de las dos series bajo estudio una vez y posteriormente aplicar la prueba ADF, especificándola de hasta 5 rezagos, con tendencia e intercepto para ambas series; se encontró que las nuevas series diferenciadas rechazaban la hipótesis nula de existencia de raíz unitaria y, por lo tanto, se concluye que la serie de primera diferencia de cada una de las series bajo estudio es estacionaria (Véase Anexo 3). Ello quiere decir que cada serie bajo estudio es integrada de orden 1: I (1). Un modelo VEC con k variables endógenas, en nuestro caso 2, debe necesariamente tener k-1 relaciones de cointegración, en nuestro caso 1 (EViews, 2018). Por lo tanto, el modelo está bien especificado.

\subsection{Criterio de información de Akaike (AIC)}

Para seleccionar el número de rezagos óptimos del modelo VEC a estimar, es aconsejable recurrir al criterio de información de Akaike, el cual estima cuanta información un proceso estocástico provee en comparación a otros y permite elegir cual da más información (Novales, 2014). Para hallar el número de rezagos, se corre un modelo VAR, sin constante, a pesar de que ese tipo de modelo econométrico requiere que las series sean estacionarias. El numero óptimo de rezagos en los modelos VEC es una unidad menor al del modelo VAR, para las mismas variables endógenas. En nuestro caso, al aplicar el AIC con 3, 4, 5, 6 y hasta 10 rezagos al modelo VAR, resulta que el número óptimo de rezagos es dos: VAR (3) (Ver Anexo 4), por lo tanto, el modelo VEC a estimar debe tener solo dos rezagos: VEC (2).

\subsection{Test de cointegración de Johansen}

La prueba de cointegración de Johansen, estima si un par o más de series de tiempo, están cointegradas entre sí. Esta prueba puede tener muchas hipótesis nulas, una para cada nivel de cointegración possible, cada una afirma la no existencia de dicha relación de cointegración para ese nivel (EViews, 2018). En nuestro caso, dado que una serie presenta una tendencia estocástica y la otra determinística, la prueba de Johansen debe llevarse a cabo asumiendo tendencia determinística, intercepto y tendencia en la única ecuación de cointegración y sin tendencia en el VAR (EViews, 2018). Al aplicar la prueba, no se rechaza la hipótesis nula, a un 
nivel de significancia del 5\%, de que existe como máximo una ecuación de cointegración. Consecuentemente, entre las dos variables, existe una relación de cointegración (Véase Anexo 5.). Ello es ideal, porque así se garantiza que el rango de cointegración de las series diferenciadas no es cero (Enders, 2003).

\subsection{Modelo VEC Estimado}

Luego de haber verificado que las dos series bajo estudio no son estacionarias, tienen el mismo grado de integración: I (1); y el número de óptimo de rezagos del modelo VEC, ya se puede estimar el modelo. Como una de las series presenta una tendencia estocástica y la otra determinista, el modelo VEC debe ser estimado con intercepto y tendencia para la ecuación de cointegración y con intercepto y sin tendencia para el modelo VEC (EViews, 2018). El modelo VEC (2) estimado para la serie de crecimiento del PIB per cápita (CPIBpc) y la serie de tasa de crecimiento del número de matrículas en educación superior (TCMes) es (Véase Anexo 6.):

$\triangle$ CPIBPc $_{1, t}=-0.4\left(\right.$ CPIBpc $_{t-1}-39.5$ TCMes $_{t-1}-0.064$ tiempo $\left.^{2} 2.26\right)-$

$0.18 \Delta C P I B p c_{1, t-1}+0.01 \Delta$ CPIBpc $_{1, t-2}-16.9 \Delta$ TCMes $_{, t-1}-16.1 \Delta$ TCMes $_{, t-2}-0.18+\varepsilon_{1 t}$

(Ecuación 10).

$\Delta$ TCMes $_{, t}=0.01\left(\right.$ CPIBpc $_{t-1}-39.5$ TCMes $_{t-1}-0.06$ tiempo +2.26$)-0.01 \Delta$ CPIBpc $_{1, t-1}-$

$0.01 \Delta$ CPIBpc $_{t-1}-0.01 \Delta$ CPIBes $_{t-2}-0.244 \Delta$ TCMes $_{t-1}-0.098 \Delta$ TCMes $\left.\left._{t-2}\right)-0.003\right)+\varepsilon_{2, t}$

(Ecuación 11).

La ecuación de cointegración estimada es representada en la Grafica 3.:

$\widehat{C P I B} c_{t-1}=-39.5$ CMMes $_{t-1}-0.06$ tiempo -2.26 (Ecuación 12).

\section{Grafica 3.}

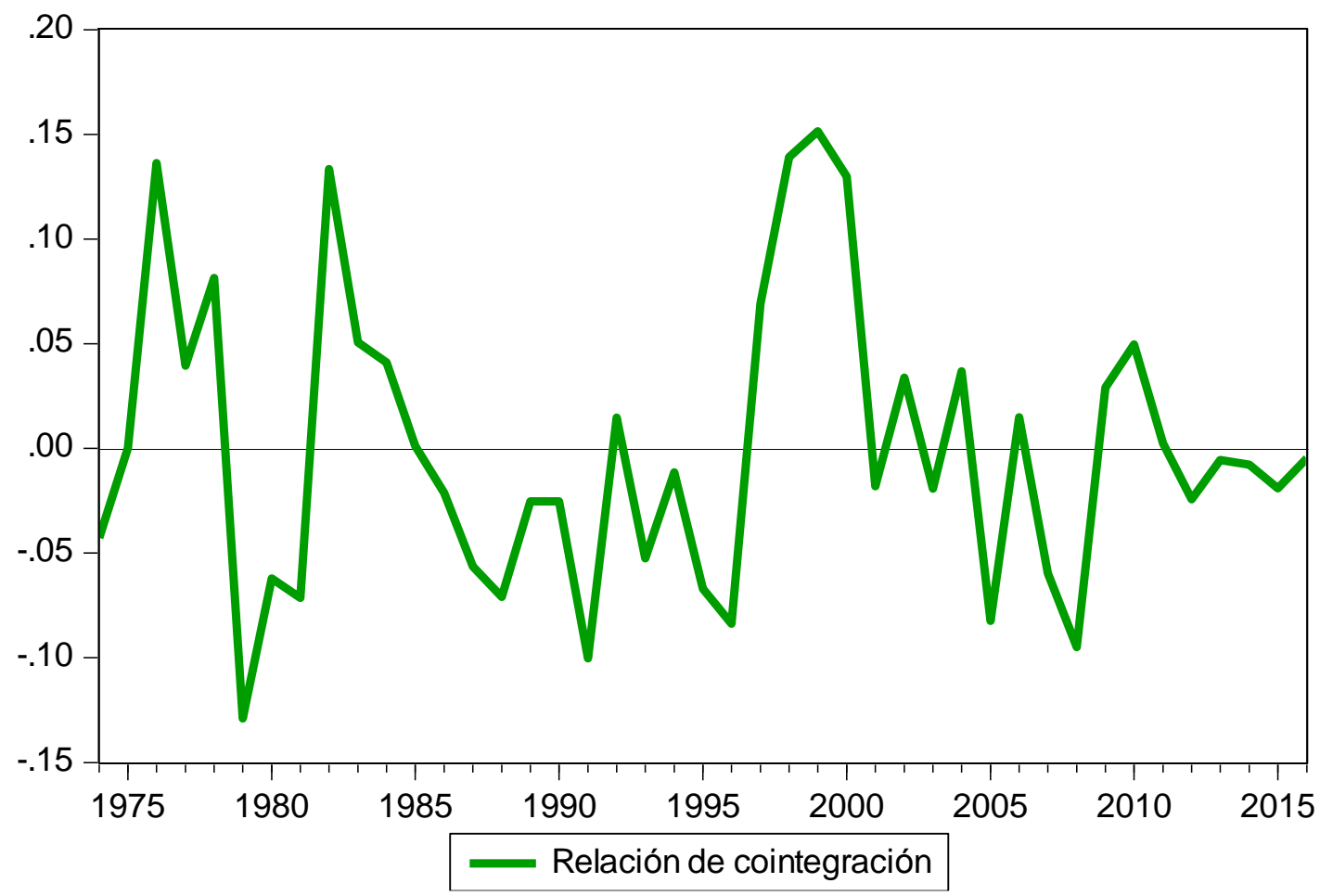

Fuente: Banco Mundial (2018). Estimación realizada en EViews. 


\section{Supuestos del modelo VEC}

Los parámetros y coeficiente $\mathrm{R}^{2}$ estimados en los modelos VAR, no son usualmente interpretados (Sosa, 2016). Sin embargo, deben verificarse el cumplimiento de ciertos supuestos, para garantizar que el modelo VEC no sea una regresión espuria, sino que en verdad refleje una relación de largo plazo implícita entre TCMes y CPIBpc. A continuación, se verificará el cumplimiento de dichos supuestos:

\subsection{No autocorrelación}

Para verificar el supuesto de no autocorrelación de los errores, observamos gráficamente los correlogramas de los residuos, hasta 10 rezagos, siguiendo a Hyndman \& Athanasopoulos (2018), de las ecuaciones de nuestro modelo estimado. Al hacerlo encontramos que parece haber autocorrelación en el octavo y noveno rezagos, en las ecuaciones 4 y 5 (Ver Anexo 6). Según Lütkepohl (2005), la prueba de Portmanteau (Prueba de Ljung-Box) para hallar autocorrelación es más robusta en la medida en que aumenta el número de rezagos, en comparación con la prueba LM, la cual es aconsejable solo para un número pequeño de rezagos (hasta 4) (Lütkepohl,2005). Así que, al aplicar la prueba de Portmanteau hasta el máximo rezago posible (n-k), 42, no se encuentra evidencia estadística significativa, para rechazar la hipótesis nula de no autocorrelación a un nivel de significancia del 5\%(Véase Anexo 7). Sin embargo, al también realizar la prueba de autocorrelación serial de los errores de los multiplicadores de Lagrange (Breusch-Pagan), hasta el rezago 15, tampoco se encontró presencia de autocorrelación, por lo que aseveramos que el modelo VEC estimado cumple el supuesto (Véase Anexo 8).

\subsection{Estabilidad del Modelo VEC}

Para verificar el supuesto de estabilidad del modelo VEC a lo largo del tiempo, a través de las raíces inversas de la prueba del polinomio característico. Se debe saber que en un modelo VEC, solamente (k-r) raíces del polinomio característico pueden ser iguales a uno, donde $\mathrm{k}$ es el número de variables endógenas y r el número de relaciones de cointegración (EViews, 2018). En nuestro modelo estimado (k-r) es igual a uno, ya que tenemos dos variables endógenas (CPIBpc y TCMes) y una sola relación de cointegración. Al realizar la prueba de las raíces del polinomio característico, se encuentra que solo la primera es igual a uno, por lo tanto, el modelo VECM cumple con el supuesto $\alpha: 0.05$ (Véase Anexo 9)

Otros de los dos supuestos del modelo VEC son que los residuos están distribuidos normalmente y que su varianza a lo largo del tiempo se mantiene constante (homocedasticidad).

Al realizar las pruebas de normalidad (según el criterio de ortogonalización de Cholesky (Lutkepohl)), se observa que el modelo cumple a un nivel de significancia de $\alpha$ : 0.05 , con el supuesto de distribucion normal de los residuos, tanto en cada uno de sus componentes, como en conjunto (Véase Anexo 10).

Al realizar las pruebas de homocedasticidad de White con términos sin cruzary cruzados, se encontró que se aceptaban las hipótesis nulas a un nivel de significancia $\alpha: 0.05$, por lo que se puede afirmar que el modelo VEC estimado cumple con el supuesto de homocedasticidad de los errores (Véase Anexo 11). 


\section{Causalidad en el sentido de Granger}

En un modelo espurio, las variables se usan para hacer inferencia estadística, como si existiera una correlación significativa entre ellas, aunque no sea así y en realidad sean independientes la una de la otra. Dado que un modelo de ese tipo no es deseable y debe evitarse, es necesario un criterio de evaluación para determinar su existencia o no, en el modelo VEC, dicho criterio es el test de casualidad de Granger (Novales, 2014).

La variable $X_{t}$ causa en el sentido de Granger a la variable $Y_{t}$ si los valores pasados de $X_{t}$ : $\left(X_{t-1}, X_{t-2} \ldots X_{t-n}\right)$, preceden y permiten predecir (temporalmente) a los valores futuros de $Y_{t}$, $\left(Y_{t+1}, Y_{t+2} \ldots Y_{t+n+1}\right)$. La prueba de causalidad de Granger permite determinar si la capacidad de predicción de los valores pasados de $\mathrm{X}_{\mathrm{t}}$ es estadísticamente significativa o no, a un nivel de significancia $\alpha$ (Novales, 2014).

La hipótesis nula de la prueba de causalidad de Granger establece que $\mathrm{X}_{\mathrm{t}}$ no causa en el sentido de Granger a $Y_{t}$. La alternativa, que $X_{t}$ causa a $Y_{t}($ Lin, 2008). Por lo tanto, realizar el test de Granger en el modelo VEC Estimado, nos ayudara a determinar si la tasa de crecimiento del número de matrículas en educación superior causo en el sentido de Granger, al crecimiento económico per capita y/o el crecimiento económico per capita causo al crecimiento del número de matrículas en educación superior, en el periodo 1971-2016 (Tabla 2):

\section{Tabla 2. Prueba de causalidad de Granger}

VEG-Granger Causality/Block Exogeneity Wald Tests

Date: 08/18/18 Time: 11:30

Sample: 19712016

Included observations: 43

Dependent variable: D(CRECIMIENTO_DEL PIB PER CAP...

\begin{tabular}{cccc}
\hline \hline Excluded & Chi-sq & df & Prob. \\
\hline \hline D(TASA_DE_CRECI... & 6.819101 & 2 & 0.0331 \\
\hline \hline All & 6.819101 & 2 & 0.0331 \\
\hline \hline
\end{tabular}

Dependent variable: D(TASA DE CRECIMIENTO DEL NU...

\begin{tabular}{cccc}
\hline \hline Excluded & Chi-sq & df & Prob. \\
\hline \hline D(CRECIMIENTO_D... & 11.68802 & 2 & 0.0029 \\
\hline \hline All & 11.68802 & 2 & 0.0029 \\
\hline
\end{tabular}

\section{Fuente: Banco Mundial (2018). Estimación realizada en EViews.}

Por un lado, como el valor p (0.0331), en la primera regression es menor a $\alpha=0.05$, es possible afirmar que la primera diferencia (variación de un año t- 1 a otro $t$ ) de la tasa de crecimiento del número de matrícula en educación superior causo en el sentido de Granger a la primera diferencia (variación de un año t-1 a otro t) del crecimiento del PIB per capita, en el periodo 1971-2016. En otras palabras, variaciones de la TCMes explican, al menos parcialmente y en un sentido temporal las variaciones del CPIBpc, en el periodo 1971-2016. 
Por el otro lado, como también el valor p (0.0029), en la segunda regression es menor a $\alpha=$ 0.05 , por lo que es posible afirmar que la primera diferencia (variación de un año t-1 a otro t) de la tasa de crecimiento del PIB per capita causo en el sentido de Granger a la primera diferencia (variación de un año t-1 a otro t) de la tasa crecimiento del número de matrícula en educación superior, en el periodo 1971-2016. Es decir, hubo una causalidad bidireccional.

En otras palabras, variaciones de la TCMes explican, al menos parcialmente y en un sentido temporal las variaciones del CPIBpc y viceversa, en el periodo 1971-2016.

\section{Funciones impulso- respuesta}

Los residuos del modelo vez: $\varepsilon_{1 t}$ and $\varepsilon_{2 t}$, pueden entenderse como desviaciones (impulsos, choques aleatorios o innovaciones)) de cada una de las variables endógenas respecto a una estimación perfecta (Stock \& Watson, 2001). Las funciones impulso-respuesta del VEC expresan como el comportamiento de una variable cambia contra un choque simulado (innovación) de los residuales de lis residuos de las demás variables endógenas o de las suyos propios. Se supone que todos los errores son iguales a cero y que un error determinado del modelo VEC estimado (choque al eatorio) regresa a cero luego de su ocurrencia, en el largo plazo (Stock \& Watson, 2001). En este caso, suponemos respuestas hasta 20 periodos luego de la ocurrencia del choque aleatorio, usando el método de descomposición de Cholesky (DOF ajustado), con innovaciones iguales a una desviación estándar:

\section{Grafica 4. Funciones impulso-respuesta}

Respuesta de TCMes a una innovación de una D.E (Cholesky d.f. adjusted)

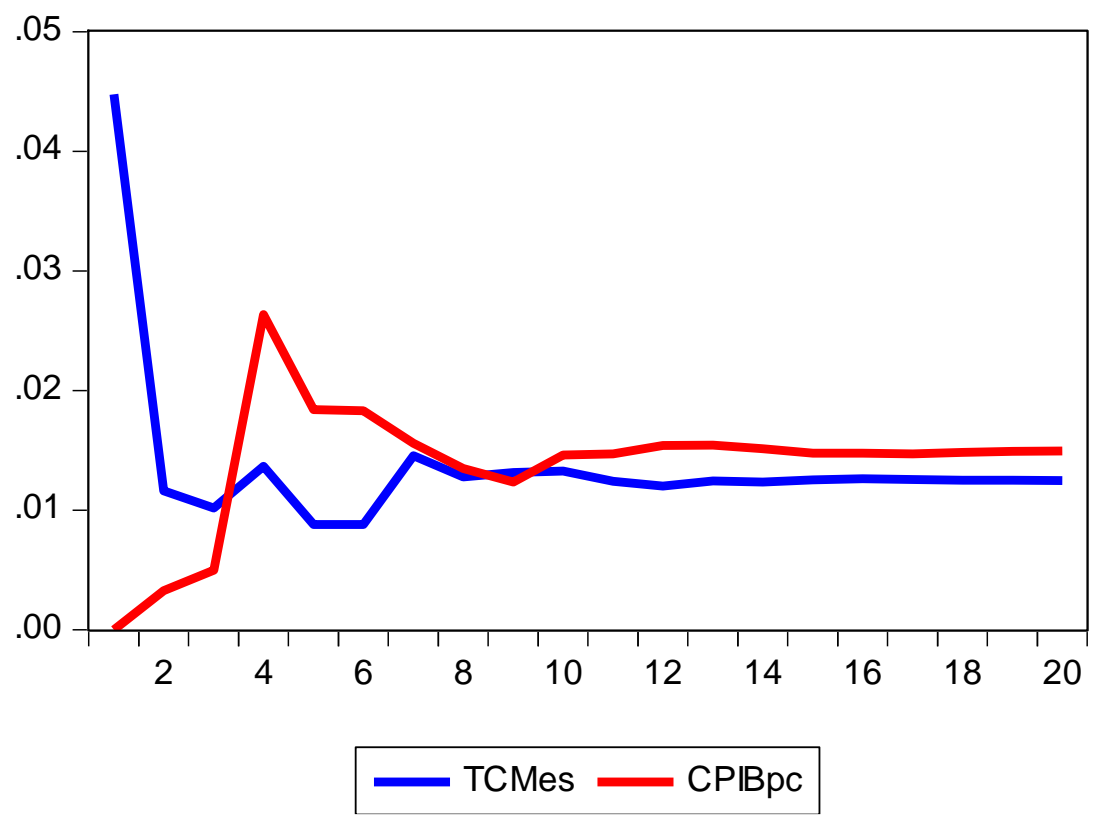

\section{Fuente: Banco Mundial (2018). Estimación realizada en EViews.}

La grafica 4 muestra que el efecto de un incremento inesperado de una desviación estándar en el crecimiento del ingreso per capita tiene un efecto permanente en la TCMes, lo cual tiene sentido, pues al aumentar el ingreso, por el principio de proporción marginal a consumir, una 
mayor parte del aumento del ingreso será destinada al ahorro, que en este caso puede ser visto como una mayor inversión en educación.

Por otro lado, un incremento o innovación de la TCMes, tiene un efecto positivo inmediato, sobre sí misma, pero en los periodos posteriores, este efecto se mitiga y estabiliza, extendiéndose a los periodos siguientes, es decir el largo plazo, afectando permanentemente la TCMes. Ello se puede explicar porque los aumentos en la capacidad instalada destinada a prestar servicios educativos no aumentan tan rápido, como la TCMes, por lo que la productividad marginal de dicho capital disminuye. Además, en general, luego de que un individuo haya adquirido un nivel educativo más alto, es menos probable que se matricule en el mismo, generalmente va a buscar uno mayor, además dado que los costos monetarios y de oportunidad aumentan, al avanzar en la escala educativa, es menos probable que pueda o quiere seguir estudiando. Otra posible razón seria, el aumento del valor agregado promedio en la economía, debido al avance técnico y desarrollo de los mercados, ello hace que haya una mayor demanda de capital humano cualificado.

\section{Grafica 5. Funciones impulse respuesta}

\section{Respuesta de CPIBPC a una innovación de una D.E (Cholesky d.f. adjusted)}

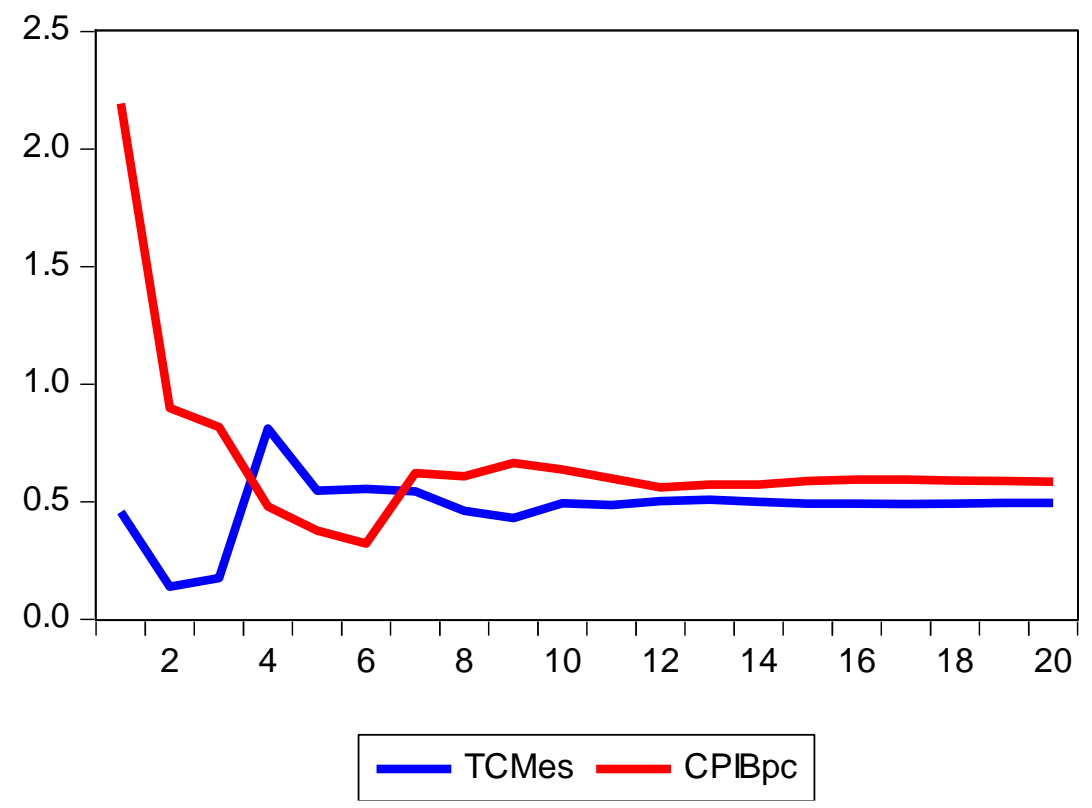

\section{Fuente: Banco Mundial (2018). Estimación realizada en EViews.}

La grafica 5, muestra las respuestas de la variable CPIBpc ante choques aleatorios o innovaciones de la variable TCMes y de ella misma, a lo largo de 20 periodos.

La respuesta del crecimiento del ingreso per capita ante un aumento de la TCMes, es positiva, pero entre el rezago 2 y el 4, fluctúa alrededor de su media. Ello puede explicarse por la duración de los programas de educación terciaria, el nivel de maestría usualmente dura dos años y el de 
licenciatura o pregrado, dura 4 años, momento en el cual el CPIBpc alcanza su máximo y desde donde los efectos del choque se esparcen a los demás periodos, volviéndose permanente.

La respuesta del crecimiento del ingreso per capita ante sí misma, es acumulativa, posiblemente porque al aumentar el ingreso, se destina una mayor parte al ahorro, en este caso educación o porque se invierte en otros activos como financieros o de otros tipos, que al generar un rendimiento refuerzan el crecimiento del ingreso mismo, etc. Dicho efecto, tiende a estabilizarse luego del cuarto periodo y a perdurar en el tiempo.

Las respuestas de cada una de las variables a cada una de las otras, es creciente y acumulativa, esto puede entenderse porque la función de capital humano: G(E), es tal como lo supone Romer (2002) es como mínimo de rendimientos constantes a escala, pero su productividad marginal no es decreciente: $G^{\prime}(E)>0$ y $G^{\prime \prime}(E)>0$, pues a medida que los individuos adquieren más educación, su capacidad para adquirir aún más aumenta también, es decir aprenden a aprender aprendiendo. Romer (2002), argumenta que en los primeros años se aprende lo básico, herramientas que no aportan mucho, porque son esenciales, pero con ellas se pueden aprender otras más complejas que si tienen un aporte mayor.

"Los datos microeconómicos sugieren que cada año adicional de que se invierte en educación incrementa el salario del trabajador aproximadamente en la misma proporción" (Romer, 2002, p.137)

Dicho efecto acumulativo a lo largo de los 20 periodos puede verse en la Grafica 6:

\section{Grafica 6. Funciones impulso respuesta}

\section{Respuesta acumulada de CPIBpc a TCMes (Cholesky one S.D d.f)}

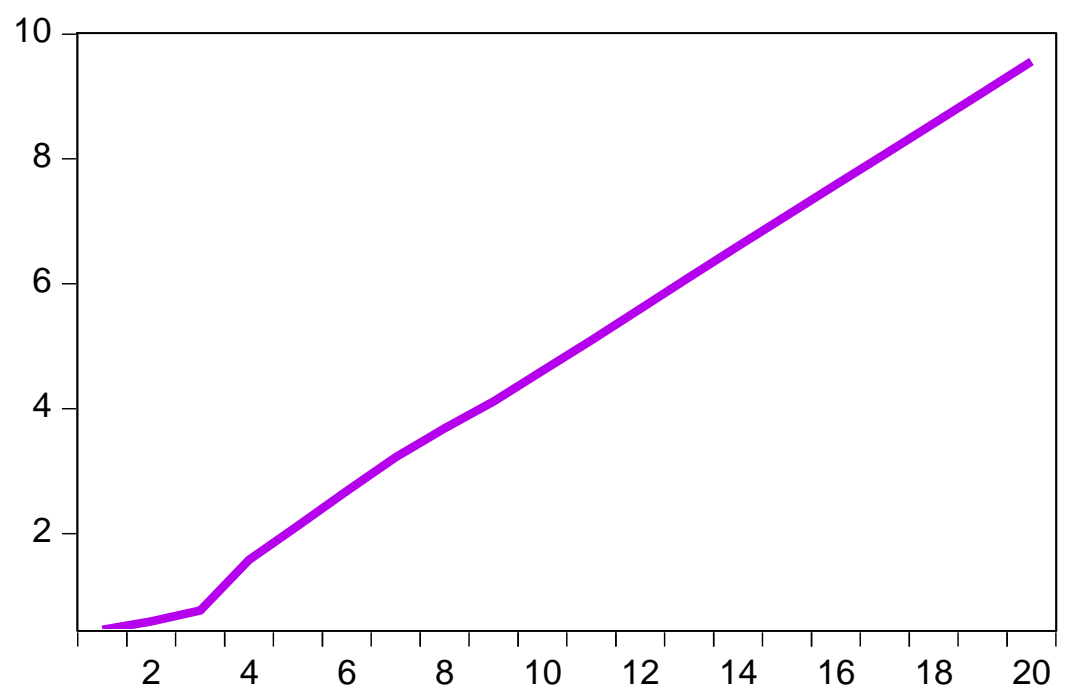

Fuente: Banco Mundial (2018). Estimación realizada en EViews.

\section{Descomposición de Varianza}

Según Novales (2014), la descomposición de varianza ayuda a estimar el tamaño del error de la predicción de cada variable, en términos de ella misma y de las demás. Los errores 
inevitablemente aumentan, al ser más largo el horizonte de predicción. Por lo tanto, la descomposición de varianza es una forma de hacer inferencia sobre las relaciones Inter temporales entre las variables endógenas del modelo VEC Estimado, ya que cada una de ellas se expresa en términos porcentuales de las varianzas de las demás (Véase Grafica 8 and Grafica 9.).

\section{Grafica 7.}

\section{Descompocision de varianza de TCMes (Cholesky (d.f. adjusted) Factors)}

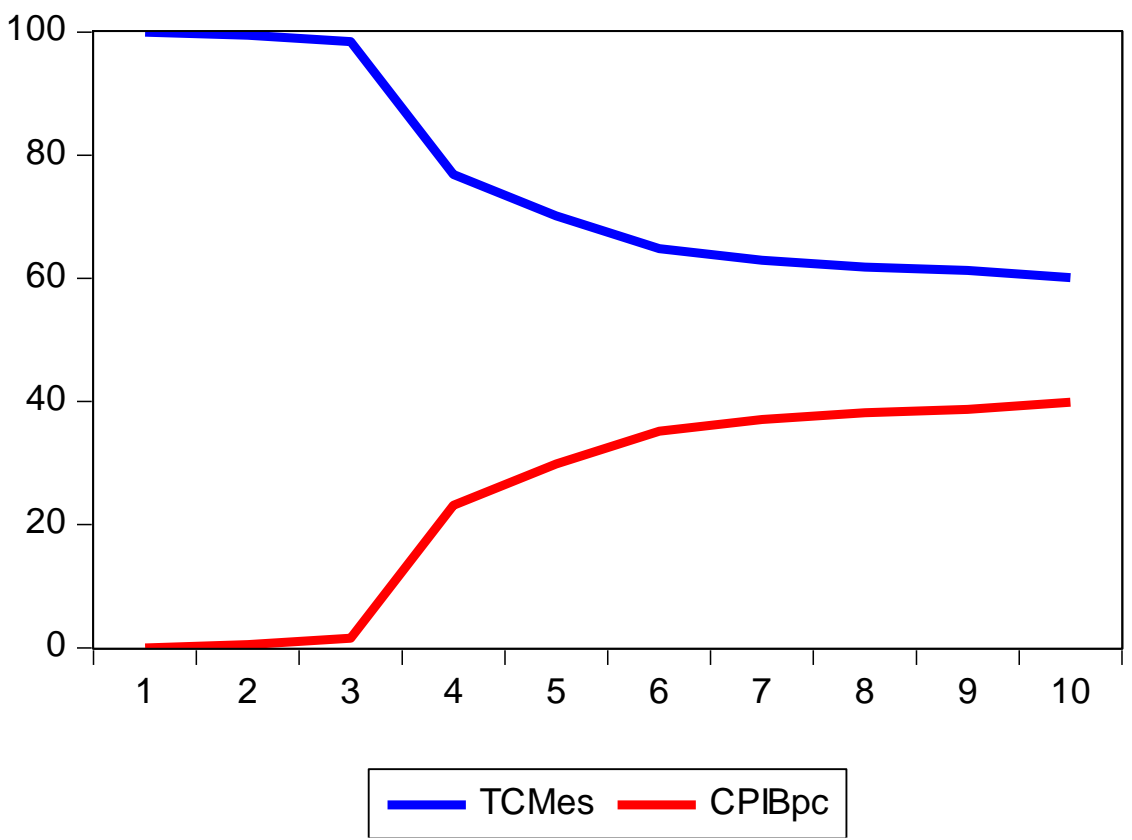

Fuente: Banco Mundial (2018). Estimación realizada en EViews.

La varianza de la TCMes (Grafica 7.) en los primeros cuatro periodos es explicada completamente por sí misma, luego en los periodos posteriores el crecimiento del ingreso per capita pasa a explicar aproximadamente el $40 \%$ de las variaciones de TCMes, teniendo un efecto permanente, en el largo plazo. Lo más probable es que esto se explique por la duración promedio de las licenciaturas y los doctorados, niveles de estudio que pertenecen a la categoría de educación superior. La varianza del CPIBpc muestra el mismo comportamiento, despega luego del cuarto periodo y desde allí se estabiliza y es explicada en un $20 \%$ por la varianza de la TCMes y en un $80 \%$ por la suya propia, es decir por el efecto acumulado de los rendimientos en inversiones de otros activos, ya sea educación, financieros, etc. (Véase Grafica 8): 


\section{Grafica 8.}

Descompocision de varianza de CPIBpc (Cholesky (d.f. adjusted) Factors)

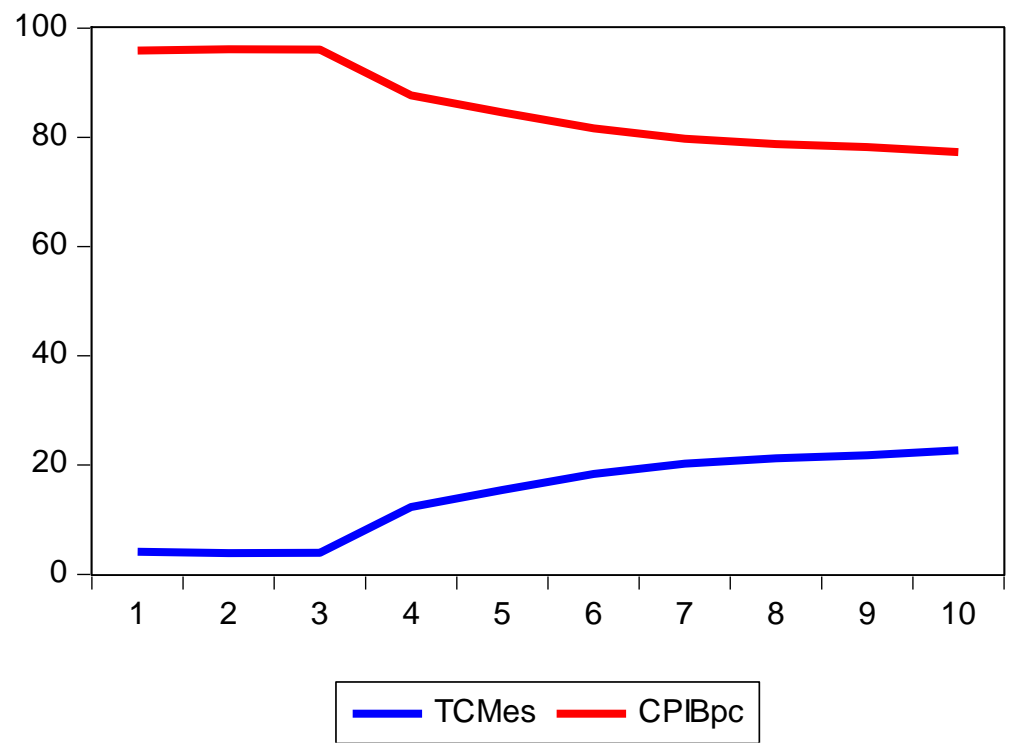

Fuente: Banco Mundial (2018). Estimación realizada en EViews.

\section{Predicción}

Aplicando un proceso de predicción dinámico al modelo VEC Estimado, para el periodo fuera de la muestra: 2017-2027, incluyendo los valores reales de las observaciones de la muestra y un coeficiente de incertidumbre de errores estándar (para reducir la mayor Fuente de error en la predicción, dado que los residuales de las predicciones no son conocidos) se obtienen las predicciones de ambas series: CPIBpc Y TCMes, para el periodo 2017-2027:

\section{Grafica 9. Predicciones de TCMes y CPIBpc}

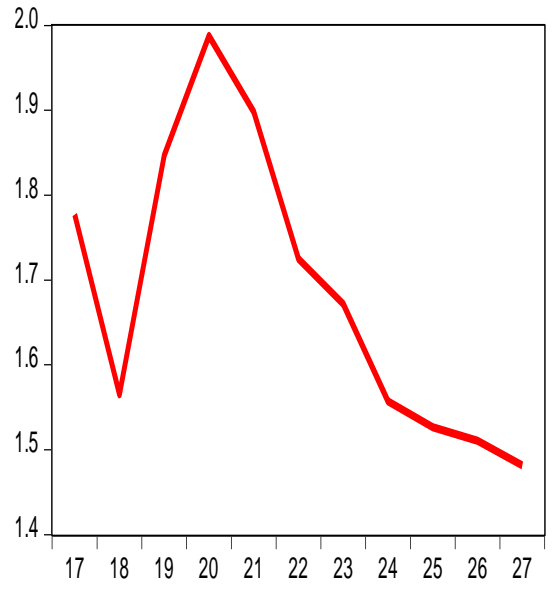

CPIBpC_F

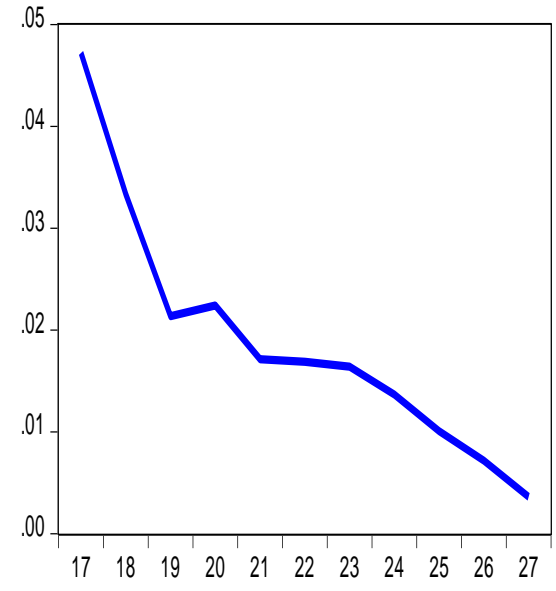

TCMes_F

Fuente: World Bank. Estimación hecha en EViews. 
Indicadores como: RMSE, MAE, MAPE e índice de Theil; son una forma d evaluar la idoneidad del modelo VEC Estimado. Una forma simple de entender su significado es que entre más cercanos a cero sean, mejores predicciones el modelo estimado produce (Woschnagg \& Cipan, 2004):

\section{Table 3. Evaluación de predicción.}

Forecast Evaluation

Date: 08/18/18 Time: 13:19

Sample: 19712016

Included observations: 46

\begin{tabular}{lccccc}
\hline \hline Variable & Inc. obs. & RMSE & MAE & MAPE & Theil \\
\hline \hline CRECIMIENT... & 46 & 2.486201 & 1.881385 & 63.29918 & 0.412895 \\
TASA_DE_CR... & 46 & 0.055840 & 0.046451 & 49.51373 & 0.299665 \\
\hline \hline
\end{tabular}

RMSE: Root Mean Square Error

MAE: Mean Absolute Error

MAPE: Mean Absolute Percentage Error

Theil: Theil inequality coefficient

\section{Fuente: World Bank. Estimación hecha en EViews.}

El índice de Theil es una buena guía para saber qué tan buenas son las predicciones del modelo, entre más cercano a cero sea, mayor es su habilidad de predicción y entre más cercano sea a uno, peores predicciones harán (Woschnagg \& Cipan, 2004). En el modelo VEC Estimado (Ver Tabla 2), el índice de Theil de la variable TCMes (Ecuación 10) es 0.29, por lo que la capacidad de predicción de esta ecuación es mejor que la del CPIBpc, sin embargo, la capacidad de predicción de esta última es aceptable, ya que su Theil es de solo 0.41 (Tabla 2).

Las funciones impulso-respuesta, la descomposición de varianza y el proceso de predicción, permiten afirmar que existe evidencia estadística suficiente para afirmar que en el periodo 1971-2016, hubo una relación bidireccional entre CPIBpc y la TCMes, es decir una relación de largo plazo, donde las innovaciones de unas variables tienen efectos permanentes y acumulativos en las trayectorias de la otra.

Como se dijo al inicio de este artículo, el crecimiento del PIB per capita, no solo depende de la acumulación de capital humano, años de educación, etc., sino que También existen otras variables. Ellas no han sido tenidas en cuenta dentro del modelo por simplicidad y porque el objetivo de este trabajo era hacer un ejercicio de dinámica comparativa, observando y analizado la relación entre crecimiento del PIB per capita y la cobertura en educación, vista desde las variaciones en el número de matrículas en educación superior, en el largo plazo. Además, al no tenerlas en cuenta, se evita perder grados de libertad, haciendo el modelo más parsimonioso.

\section{Discusión and conclusiones:}

Ahora, somos capaces de responder a la pregunta con la que inicio este texto: ¿Qué tipo de relación existió entre Crecimiento del PIB per capita y la cobertura en educación, vista desde el 
número de matrículas en educación superior, en el period 1971-2016? El modelo econométrico ayuda a responder esta pregunta: Existió efectivamente una relación de cointegración o de largo plazo, entre CPIBpc y TCMes, en el largo plazo. Posteriormente, se comprobó que CPIBpc causo en el sentido de Granger a TCMes y viceversa, TCMes Granger-causo a su vez también al CPIBpc. Es decir, existió una relación de causalidad temporal entre ambas variables, cuyos efectos fueron permanentes, se extendían y acumulaban en el tiempo.

Dado que la relación entre las dos variables fue bidireccional, una explicaba, al menos parcial y temporalmente, a la otra, la elección del modelo VEC fue apropiada, pues al tratar con las dos variables como endógenas al mismo tiempo, se maneja mejor empíricamente el problema de endogeneidad.

No solo se trata de aumentar los años de escolaridad, sino de mejorar las habilidades cognitivas y acervo de conocimiento de la mayor cantidad de individuos posibles en la población. Para ello, no solo basta con abrir más colegios, universidades, institutos y programas, sino también acompañar y apoyar en los procesos de aprendizaje a los estudiantes, desde el punto de vista nutricional, de salud, psicológico y otras variables que influyen en su desempeño escolar y laboral futuro y en su capacidad de aprendizaje. Ojalá desde la primera infancia (Hanushek, 2016). Es por eso que programas como 'De cero a siempre' que el Estado colombiano lleva implementando desde el año 2016 (Gobierno de Colombia, 2018), tienen un potencial tan alto, ya que no solo velan por los derechos de los niños hoy, sino que haciéndolo hoy, oportunamente a edades tan sensibles y vitales en su desarrollo mental, físico, psicológico, etc., contribuyen en gran medida a hacerlo también en el futuro. Sus efectos se esparcen y acumulan en forman de capital humano en el tiempo y por lo tanto afectan el crecimiento del ingreso per capita.

\section{Referencias bibliograficas.}

Barro, R. J. (2001). Educationand economic growth. The contribution of human and social capital to sustained economic growth and well-being, 14-41.

Enders, W. (2003). Applied Econometric Time Series. John Wiley \& Sons.

EViews 10 Help Topics. (2018). Retrieved from: http://www.eviews.com/help/helpintro.html

Gobierno de Colombia., (2018)., Descripción del programa 'De cero a siempre' . Recuperado el 24 de agosto de 2018 de: http://www.deceroasiempre.gov.co/QuienesSomos/Paginas/QuienesSomos.aspx

Hamilton, J. D. (1994). Time series analysis (Vol. 2). Princeton: Princeton university press.

Hanushek, E. A. (2013). Economic growth in developing countries: The role of human capital. Economics of Education Review, 37, 204-212.

Hanushek, E. A. (2016). Will more higher education improve economic growth?. Oxford Review of Economic Policy, 32(4), 538-552.

Hyndman, R. J., \& Athanasopoulos, G. (2018). Forecasting: principles and practice. OTexts.

Lin, J. (2008). Notes on testing causality. Institute of Economics, Academia Sinica, Department of Economics, National Chengchi University. 
Lütkepohl, H. (2005). New introduction to multiple time series analysis. Springer Science \& Business Media.

Mercan, M., \& Sezer, S. (2014). The effect of education expenditure on economic growth: The case of Turkey. Procedia-Social and Behavioral Sciences, 109(0), 925-930.

Novales, A. (2014). Modelos vectoriales autoregresivos (VAR).

Romer, D. (2012). Advanced macroeconomics. Fourth edition. Mac Graw Hill.

Sala-i-Martin, X. (2000). Apuntes de crecimiento económico. Antoni Bosch Editor.

Sosa, W. (2006). Vectores autoregresivos (VAR), curso del Banco central de Chile: Vectores Autorregresivos para Datos en Paneles. Retrieved on November 21 of 2016 from: http://faculty.udesa.edu.ar/WalterSosa/PVAR/bcchile2.pdf

Stock, J. H., \& Watson, M. W. (2001). Vector autoregressions. The Journal of Economic Perspectives, 15(4), 101-115.

The World Bank. (2018). Database: World Development Indicators. Retrieved from http://databank.worldbank.org/data/reports.aspx?source=2\&country=COL\#

Woschnagg, E \& Cipan, J. (2004). Evaluating forecast accuracy. University of Vienna, Department of Economics. 


\section{Anexo 1.}

Null Hypothesis: CRECIMIENTO_DEL_PIB_PER_CAPITA_ANUAL_ has a unit root Exogenous: Constant, Linear Trend Lag Length: 5 (Fixed)

\begin{tabular}{llcc}
\hline \hline & & t-Statistic & Prob. $^{*}$ \\
\hline \hline Augmented Dickev-Fuller test statistic & & -2.266487 & 0.4417 \\
\hline Test critical values: & $1 \%$ level & -4.205004 & \\
& $5 \%$ level & -3.526609 & \\
& $10 \%$ level & -3.194611 & \\
\hline \hline
\end{tabular}

*MacKinnon (1996) one-sided p-values.

Augmented Dickey-Fuller Test Equation

Dependent Variable: D(CRECIMIENTO_DEL_PIB_PER_CAPITA AN

UAL_)

Method: Least Square

Date: 08/16/18 Time: $13: 31$

Included observations: 40 after adjustments

\begin{tabular}{lrrrr}
\hline \multicolumn{1}{c}{ Variable } & Coefficient & Std. Error & t-Statistic & Prob. \\
\hline \hline CRECIMIENTO_DEL_PIB_PER_CAPIT... & -0.729890 & 0.322036 & -2.266487 & 0.0303 \\
D(CRECIMIENTO_DEL_PIB_PER_CAP... & -0.004372 & 0.290177 & -0.015065 & 0.9881 \\
D(CRECIMIENTO_DEL_PIB_PER_CAP... & 0.109819 & 0.253728 & 0.432821 & 0.6680 \\
D(CRECIMIENTO_DEL_PIB PER CAP... & 0.105892 & 0.228586 & 0.463247 & 0.6463 \\
D(CRECIMIENTO_DEL_PIB_PER_CAP... & -0.056014 & 0.205649 & -0.272376 & 0.7871 \\
D(CRECIMIENTO_DEL_PIB_PER_CAP... & -0.183794 & 0.169364 & -1.085200 & 0.2859 \\
C & 0.853828 & 0.995035 & 0.858089 & 0.3972 \\
\multicolumn{1}{c}{ @TREND("1971") } & 0.026429 & 0.031524 & 0.838398 & 0.4080 \\
\hline \hline R-squared & 0.405487 & Mean dependent var & -0.033009 \\
Adjusted R-squared & 0.275437 & S.D. dependent var & 2.578723 \\
S.E. of regression & 2.195042 & Akaike info criterion & 4.587136 \\
Sum squared resid & 154.1827 & Schwarz criterion & 4.924912 \\
Log likelihood & -83.74272 & Hannan-Quinn criter. & 4.709265 \\
F-statistic & 3.117935 & Durbin-Watson stat & 1.970275 \\
Prob(F-statistic) & 0.012637 & & & \\
\hline
\end{tabular}

\section{Anexo 2.}

Null Hypothesis: TASA DE_CRECIMIENTO_DEL_NUMERO_DE_MATRICULAS_EN_ED... Exogenous: Constant, Linear Trend Lag Length: 5 (Fixed)

\begin{tabular}{llrc}
\hline \hline & & t-Statistic & Prob. $^{*}$ \\
\hline \hline Augmented Dickev-Fuller test statistic & & -2.679997 & 0.2498 \\
\hline Test critical values: & $1 \%$ level & -4.205004 & \\
& $5 \%$ level & -3.526609 & \\
& $10 \%$ level & -3.194611 & \\
\hline \hline
\end{tabular}

*MacKinnon (1996) one-sided p-values.

Augmented Dickey-Fuller Test Equation

Dependent Variable: D(TASA DE_CRECIMIENTO_DEL_NUMERO_DE MATRICULAS_EN_EDUCACION_SUPERI)

Method: Least Squares

Date: 08/16/18 Time: 13:32

Sample (adjusted): 19772016

Included observations: 40 after adjustments

\begin{tabular}{lrrrr}
\multicolumn{1}{c}{ Variable } & Coefficient & Std. Error & t-Statistic & Prob. \\
\hline \hline TASA DE_CRECIMIENTO_DEL_NUM... & -0.768965 & 0.286928 & -2.679997 & 0.0115 \\
D(TASA DE_CRECIMIENTO_DEL_NU... & -0.124117 & 0.267257 & -0.464411 & 0.6455 \\
D(TASA_DE_CRECIMIENTO_DEL_NU... & -0.071878 & 0.245238 & -0.293094 & 0.7713 \\
D(TASA_DE_CRECIMIENTO_DEL_NU... & -0.092827 & 0.225615 & -0.411439 & 0.6835 \\
D(TASA_DE_CRECIMIENTO_DEL_NU... & -0.229096 & 0.196728 & -1.164533 & 0.2528 \\
D(TASA_DE_CRECIMIENTO_DEL_NU... & -0.093391 & 0.161769 & -0.577310 & 0.5678 \\
C & 0.048228 & 0.034684 & 1.390523 & 0.1740 \\
\multicolumn{1}{c}{ @TREND("1971") } & $-2.79 E-05$ & 0.000737 & -0.037942 & 0.9700 \\
\hline \hline & \multirow{2}{*}{0.483747} & Mean dependent var & -0.002640 \\
R-squared & 0.370817 & S.D. dependent var & 0.058773 \\
Adjusted R-squared & 0.046619 & Akaike info criterion & -3.116740 \\
S.E. of regression & 0.069548 & Schwarz criterion & -2.778964 \\
Sum squared resid & 70.33480 & Hannan-Quinn criter. & -2.994611 \\
Log likelihood & 4.283593 & Durbin-Watson stat & 2.040208 \\
F-statistic & 0.001935 & & & \\
Prob(F-statistic) & & & & \\
\hline
\end{tabular}




\section{Anexo 3.}

Null Hypothesis: D(CRECIMIENTO DEL PIB PER CAPITA ANUAL) has a unit root Exogenous: Constant, Linear Trend

Lag Length: 5 (Fixed)

\begin{tabular}{llcc}
\hline \hline & & t-Statistic & Prob. $^{*}$ \\
\hline \hline Augmented Dickey-Fuller test statistic & & -3.635405 & 0.0396 \\
\hline Test critical values: & $1 \%$ level & -4.211868 & \\
& $5 \%$ level & -3.529758 & \\
& $10 \%$ level & -3.196411 & \\
\hline \hline
\end{tabular}

*MacKinnon (1996) one-sided p-values.

Augmented Dickey-Fuller Test Equation

Dependent Variable: D(CRECIMIENTO_DEL_PIB_PER_CAPITA_AN

$$
\text { UAL ,2) }
$$

Method: Least Squares

Date: 08/16/18 Time: $13: 39$

Sample (adjusted): 19782016

Included observations: 39 after adjustments

\begin{tabular}{lrrrr}
\multicolumn{1}{c}{ Variable } & Coefficient & Std. Error & t-Statistic & Prob. \\
\hline \hline D(CRECIMIENTO DEL_PIB PER CAP... & -2.902920 & 0.798513 & -3.635405 & 0.0010 \\
D(CRECIMIENTO_DEL_PIB_PER_CAP... & 1.328420 & 0.695575 & 1.909817 & 0.0654 \\
D(CRECIMIENTO_DEL_PIB_PER_CAP... & 0.998292 & 0.574668 & 1.737164 & 0.0923 \\
D(CRECIMIENTO_DEL_PIB_PER_CAP... & 0.751134 & 0.454098 & 1.654123 & 0.1082 \\
D(CRECIMIENTO_DEL_PIB_PER_CAP... & 0.413248 & 0.326205 & 1.266834 & 0.2147 \\
D(CRECIMIENTO_DEL_PIB_PER_CAP... & 0.039164 & 0.178139 & 0.219852 & 0.8274 \\
C & -0.317749 & 0.993899 & -0.319700 & 0.7513 \\
\multicolumn{1}{c}{ @TREND("1971") } & 0.012228 & 0.035270 & 0.346689 & 0.7312 \\
\hline \hline & 0.753424 & Mean dependent var & -0.007772 \\
R-squared & 0.697745 & S.D. dependent var & 4.366565 \\
Adjusted R-squared & 2.400638 & Akaike info criterion & 4.770028 \\
S.E. of regression & 178.6549 & Schwarz criterion & 5.111271 \\
Sum squared resid & -85.01555 & Hannan-Quinn criter. & 4.892463 \\
Log likelihood & 13.53167 & Durbin-Watson stat & 1.925981 \\
F-statistic & 0.000000 & & & \\
Prob(F-statistic) & & & & \\
\hline
\end{tabular}

Null Hypothesis: D(TASA DE CRECIMIENTO DEL NUMERO DE MATRICULAS EN E... Exōenous: Constant, Linear Trend

Lag Length: 5 (Fixed)

\begin{tabular}{llcc}
\hline \hline & & t-Statistic & Prob. $^{*}$ \\
\hline \hline Auqmented Dickey-Fuller test statistic & & -3.757920 & 0.0300 \\
\hline Test critical values: & 1\% level & -4.211868 & \\
& $5 \%$ level & -3.529758 & \\
& $10 \%$ level & -3.196411 & \\
\hline \hline
\end{tabular}

*MacKinnon (1996) one-sided p-values.

Augmented Dickey-Fuller Test Equation

Dependent Variable: D(TASA DE_CRECIMIENTO_DEL_NUMERO_DE MATRICULAS_EN EDUCACION_SUPERI,2

Method: Least Squares

Date: 08/16/18 Time: 13:39

Sample (adjusted): 19782016

Included observations: 39 after adjustments

\begin{tabular}{lrrrr}
\multicolumn{1}{c}{ Variable } & Coefficient & Std. Error & t-Statistic & Prob. \\
\hline \hline D(TASA DE_CRECIMIENTO_DEL_NU... & -3.563625 & 0.948297 & -3.757920 & 0.0007 \\
D(TASA_DE_CRECIMIENTO_DEL_NU... & 1.844288 & 0.845082 & 2.182376 & 0.0368 \\
D(TASA_DE_CRECIMENTO_DEL_NU... & 1.269696 & 0.689059 & 1.842652 & 0.0750 \\
D(TASA_DE_CRECIMIENTO_DEL_NU... & 0.786799 & 0.525704 & 1.496658 & 0.1446 \\
D(TASA_DE_CRECIMIENTO_DEL_NU... & 0.302146 & 0.354233 & 0.852960 & 0.4002 \\
D(TASA_DE_CRECIMIENTO_DEL_NU... & 0.055842 & 0.178564 & 0.312729 & 0.7566 \\
C C & -0.036856 & 0.023021 & -1.600999 & 0.1195 \\
\multicolumn{1}{c}{ @TREND("1971") } & 0.001052 & 0.000793 & 1.327230 & 0.1941 \\
\hline \hline & 0.781904 & Mean dependent var & -0.000317 \\
R-squared & 0.732657 & S.D. dependent var & 0.100415 \\
Adjusted R-squared & 0.051920 & Akaike info criterion & -2.897553 \\
S.E. of regression & 0.083565 & Schwarz criterion & -2.556310 \\
Sum squared resid & 64.50228 & Hannan-Quinn criter. & -2.775118 \\
Log likelihood & 15.87703 & Durbin-Watson stat & 1.897761 \\
F-statistic & 0.000000 & & & \\
Prob(F-statistic) & & & & \\
\hline & & & &
\end{tabular}




\section{Anexo 4.}

VAR Lag Order Selection Criteria

Endogenous variables: CRECIMIENTO_DEL_PIB_PER_CAPITA__ANUAL_T...

Exogenous variables:

Date: 08/16/18 Time: $14: 39$

Sample: 19712016

Included observations: 36

\begin{tabular}{ccccccc}
\hline \hline Lag & LogL & LR & FPE & AIC & SC & HQ \\
\hline \hline 1 & -22.40423 & NA & 0.014867 & 1.466902 & $1.642848^{*}$ & 1.528312 \\
2 & -18.43633 & 7.054039 & 0.014917 & 1.468685 & 1.820578 & 1.591505 \\
3 & -11.53650 & $11.49972^{*}$ & $0.012754^{\star}$ & $1.307583^{*}$ & 1.835423 & $1.491814^{*}$ \\
4 & -9.454212 & 3.239116 & 0.014314 & 1.414123 & 2.117909 & 1.659763 \\
5 & -6.929929 & 3.646186 & 0.015770 & 1.496107 & 2.375840 & 1.803157 \\
6 & -5.775711 & 1.538958 & 0.018900 & 1.654206 & 2.709885 & 2.022666 \\
7 & -5.455344 & 0.391559 & 0.023976 & 1.858630 & 3.090256 & 2.288501 \\
8 & -4.101496 & 1.504276 & 0.029104 & 2.005639 & 3.413211 & 2.496919 \\
9 & -0.675318 & 3.426177 & 0.032032 & 2.037518 & 3.621037 & 2.590208 \\
10 & 3.825875 & 4.001061 & 0.033953 & 2.009674 & 3.769139 & 2.623774 \\
\hline \hline
\end{tabular}

* indicates lag order selected by the criterion

LR: sequential modified LR test statistic (each test at $5 \%$ level)

FPE: Final prediction error

AIC: Akaike information criterion

SC: Schwarz information criterion

$\mathrm{HQ}$ : Hannan-Quinn information criterion

\section{Anexo 5}

Date: $08 / 16 / 18$ Time: $14: 46$

Sample (adjusted): 19742016

Included observations: 43 after adjustments

Trend assumption: Linear deterministic trend (restricted)

Series: CRECIMIENTO_DEL_PIB_PER_CAPITA ANUAL_TASA D...

Lags interval (in first differences): 1 to 2

Unrestricted Cointegration Rank Test (Trace)

\begin{tabular}{|c|c|c|c|c|}
\hline $\begin{array}{l}\text { Hypothesized } \\
\text { No. of } C E(s)\end{array}$ & Eigenvalue & $\begin{array}{c}\text { Trace } \\
\text { Statistic }\end{array}$ & $\begin{array}{c}0.05 \\
\text { Critical Value }\end{array}$ & Prob. ${ }^{\star *}$ \\
\hline None * & 0.374586 & 28.74546 & 25.87211 & 0.0213 \\
\hline At most 1 & 0.180579 & 8.563761 & 12.51798 & 0.2090 \\
\hline \multicolumn{5}{|c|}{$\begin{array}{l}\text { Trace test indicates } 1 \text { cointegrating eqn(s) at the } 0.05 \text { level } \\
\text { * denotes rejection of the hypothesis at the } 0.05 \text { level } \\
{ }^{* *} \text { MacKinnon-Haug-Michelis (1999) p-values }\end{array}$} \\
\hline \multicolumn{5}{|c|}{ Unrestricted Cointegration Rank Test (Maximum Eigenvalue) } \\
\hline $\begin{array}{l}\text { Hypothesized } \\
\text { No. of CE(s) }\end{array}$ & Eigenvalue & $\begin{array}{l}\text { Max-Eigen } \\
\text { Statistic }\end{array}$ & $\begin{array}{l}0.05 \\
\text { Critical Value }\end{array}$ & Prob.** \\
\hline $\begin{array}{l}\text { None * } \\
\text { At most } 1\end{array}$ & $\begin{array}{l}0.374586 \\
0.180579\end{array}$ & $\begin{array}{l}20.18169 \\
8.563761\end{array}$ & $\begin{array}{l}19.38704 \\
12.51798\end{array}$ & $\begin{array}{l}0.0383 \\
0.2090\end{array}$ \\
\hline
\end{tabular}

Max-eigenvalue test indicates 1 cointegrating eqn(s) at the 0.05 level

* denotes rejection of the hypothesis at the 0.05 level

${ }^{* *}$ MacKinnon-Haug-Michelis (1999) p-values

Unrestricted Cointegrating Coefficients (normalized by $b^{* *} S 11^{*} b=I$ ):

\begin{tabular}{ccc}
\hline \hline CRECIMIEN... & TASA DE ... & @TREND(72) \\
0.526232 & -20.78710 & -0.033859 \\
0.377796 & 21.60149 & 0.002906 \\
\hline \hline
\end{tabular}

Unrestricted Adjustment Coefficients (alpha):

\begin{tabular}{|c|c|c|c|}
\hline $\begin{array}{l}\text { D(CRECIMI... } \\
\text { D(TASA_DE... }\end{array}$ & $\begin{array}{r}-0.762239 \\
0.024592\end{array}$ & $\begin{array}{l}-0.777360 \\
-0.011348 \\
\end{array}$ & \\
\hline 1 Cointegrating & Equation(s): & Log likelihood & -15.74032 \\
\hline \multicolumn{4}{|c|}{ Normalized cointegrating coefficients (standard error in parentheses) } \\
\hline $\begin{array}{c}\text { CRECIMIEN... } \\
1.000000\end{array}$ & $\begin{array}{r}\text { TASA DE ... } \\
-39.50178 \\
(12.2562)\end{array}$ & $\begin{array}{c}\text { @TREND(72) } \\
-0.064342 \\
(0.03652)\end{array}$ & \\
\hline \multicolumn{4}{|c|}{ Adjustment coefficients (standard error in parentheses) } \\
\hline D(CRECIMI... & $\begin{array}{r}-0.401115 \\
(0.17974)\end{array}$ & & \\
\hline D(TASA DE... & $\begin{array}{l}0.012941 \\
(0.00359)\end{array}$ & & \\
\hline
\end{tabular}




\section{Anexo 6.}

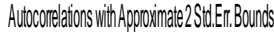

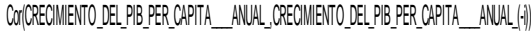

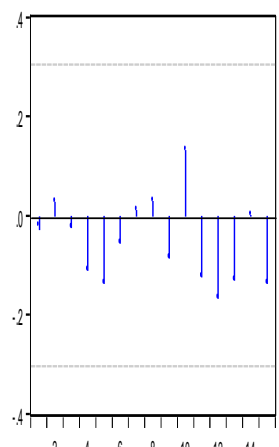

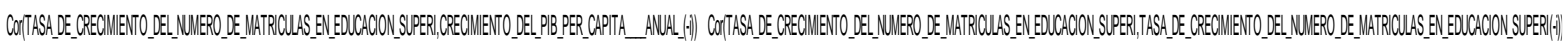

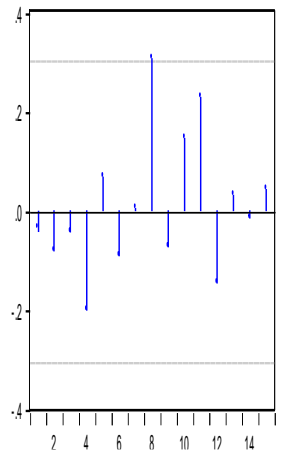

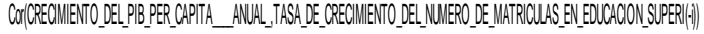

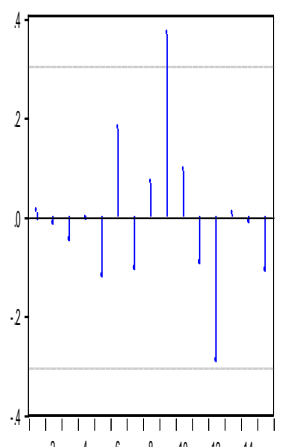

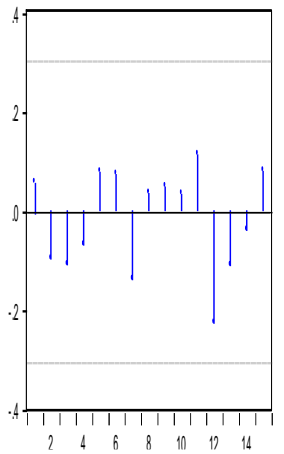




\section{Anexo 7.}

VEC Residual Portmanteau Tests for Autocorrelations
Null Hypothesis: No residual autocorrelations up to lag $\mathrm{h}$

Sample: 19712016

\begin{tabular}{|c|c|c|c|c|c|}
\hline Lags & Q-Stat & Prob. ${ }^{*}$ & Adj Q-Stat & Prob. ${ }^{*}$ & df \\
\hline 1 & 0.236851 & $\cdots$ & 0.242490 & -.. & -.. \\
\hline 2 & 911698 & -.. & 0.950257 & $\ldots$ & \\
\hline 3 & 1.502819 & 0.9822 & 1.585712 & 0.9792 & 7 \\
\hline 4 & 3.535446 & 0.9816 & 3.826814 & 0.9747 & 11 \\
\hline 5 & 5.495668 & 0.9870 & 6.044959 & 0.9790 & 15 \\
\hline 6 & 7.599209 & 0.9903 & 8.489615 & 0.9810 & 19 \\
\hline 7 & 8.812622 & 0.9966 & 9.938969 & 0.9916 & 23 \\
\hline 8 & 13.15336 & 0.9882 & 15.27187 & 0.9654 & 27 \\
\hline 9 & 20.19716 & & 24.18021 & & 31 \\
\hline 10 & 21.74757 & 0.9610 & 26.20044 & 0.8586 & 35 \\
\hline 11 & 25.81553 & 0.9481 & 31.66676 & 0.7916 & 39 \\
\hline 12 & $\begin{array}{l}31.62278 \\
33.25627\end{array}$ & $\begin{array}{l}0.9001 \\
0.9351\end{array}$ & $\begin{array}{r}39.72198 \\
\end{array}$ & 0.6143 & 43 \\
\hline $\begin{array}{l}13 \\
14\end{array}$ & $\begin{array}{l}33.25667 \\
333.33059\end{array}$ & $\begin{array}{l}0.9351 \\
0.9737\end{array}$ & $\begin{array}{r}42.06332 \\
42.17351\end{array}$ & $\begin{array}{l}0.6768 \\
0.8060\end{array}$ & $\begin{array}{l}47 \\
51\end{array}$ \\
\hline $\begin{array}{l}14 \\
15\end{array}$ & $\begin{array}{l}333.33359 \\
35.03841\end{array}$ & $\begin{array}{l}0.9737 \\
0.9836\end{array}$ & $\begin{array}{l}42.17351 \\
44.79624\end{array}$ & $\begin{array}{l}0.8060 \\
0.8354\end{array}$ & $\begin{array}{l}51 \\
55\end{array}$ \\
\hline 16 & 36.21042 & 0.9915 & $\begin{array}{l}46.19024 \\
46.66277\end{array}$ & $\begin{array}{l}0.03777 \\
0.877\end{array}$ & $\begin{array}{l}50 \\
59\end{array}$ \\
\hline 17 & 39.12341 & 0.9922 & 51.48041 & 0.8499 & 63 \\
\hline 18 & 48.52300 & 0.9567 & 67.64771 & 0.4549 & 67 \\
\hline 19 & 51.67363 & 0.9591 & 73.29259 & 0.4028 & 71 \\
\hline 20 & 52.03911 & 0.9800 & 73.9 & 0.5118 & 75 \\
\hline 21 & 53.64 & 0.9872 & 77.1 & & 79 \\
\hline 22 & 56.08318 & 0.9898 & $\begin{array}{r}82.10737 \\
87\end{array}$ & 0.5071 & 83 \\
\hline & $\begin{array}{r}58.47368 \\
\end{array}$ & $\begin{array}{l}0.9919 \\
0.9934\end{array}$ & $\begin{array}{l}87.24694 \\
\end{array}$ & $\begin{array}{l}0.4724 \\
\end{array}$ & 87 \\
\hline & $\begin{array}{l}60.99985 \\
61.30893\end{array}$ & 0.9934 & $\begin{array}{r}92.96406 \\
9370241\end{array}$ & $\begin{array}{l}0.4231 \\
0.5184\end{array}$ & $\begin{array}{l}91 \\
95\end{array}$ \\
\hline 26 & $\begin{array}{l}61.30893 \\
61.48079\end{array}$ & $\begin{array}{l}0.9971 \\
0.9989\end{array}$ & $\begin{array}{l}93.702211 \\
94.13713\end{array}$ & $\begin{array}{l}0.5184 \\
0.6194\end{array}$ & $\begin{array}{l}95 \\
99\end{array}$ \\
\hline $\begin{array}{l}26 \\
27\end{array}$ & $\begin{array}{l}61.48079 \\
61.93730\end{array}$ & $\begin{array}{l}0.99989 \\
0.9995\end{array}$ & $\begin{array}{l}94.13713 \\
95.36399\end{array}$ & $\begin{array}{l}0.6194 \\
0.6911\end{array}$ & $\begin{array}{l}99 \\
103\end{array}$ \\
\hline & 62.65705 & 0.9998 & 97.42729 & 0.7353 & 107 \\
\hline & 63.18 & 0.9999 & 99.04210 & 0.7 & 111 \\
\hline 30 & 64.84256 & 1.0000 & 104 & & 115 \\
\hline 31 & 65.92 & & 108. & & 119 \\
\hline 32 & 66.25 & 1.0000 & 109. & & 123 \\
\hline 33 & 66.85 & 1.0 & 112 & & 127 \\
\hline 34 & 67.32387 & 1.0000 & 114. & & 131 \\
\hline 35 & 67.69403 & 1.0000 & $\begin{array}{l}116.5134 \\
\end{array}$ & 0.8 & 135 \\
\hline & $\begin{array}{l}68.56317 \\
68.637\end{array}$ & $\begin{array}{l}1.0000 \\
1.0000\end{array}$ & $\begin{array}{r}121.8524 \\
122.3820\end{array}$ & $\begin{array}{c}0.8493 \\
0.8931\end{array}$ & 139 \\
\hline $\begin{array}{l}37 \\
38\end{array}$ & 68.72289 & $\begin{array}{l}1.00000 \\
1.000\end{array}$ & $\begin{array}{l}122.3880 \\
123.1200\end{array}$ & $\begin{array}{l}0.8931 \\
0.9246\end{array}$ & $\begin{array}{l}143 \\
147\end{array}$ \\
\hline 39 & 68.83 & 1.0000 & 124. & 0.9449 & 151 \\
\hline 40 & 68.90 & 1.0000 & 125.3636 & 0.9613 & 155 \\
\hline 41 & 69.01129 & 1.0000 & 127.5994 & 0.9681 & 159 \\
\hline 42 & 69.01468 & 1.0000 & 127.7452 & 0.9811 & 163 \\
\hline
\end{tabular}

"Test is valid only for lags larger than the VAR lag order.

If is degrees of freedom for (approximate) chi-square distribution
after adiustment for VEC estimation (Bruggemann, et al. 2005)

\section{Anexo 8.}




\begin{tabular}{|c|c|c|c|c|c|c|}
\hline \multicolumn{7}{|c|}{$\begin{array}{l}\text { VEC Residual Serial Correl } \\
\text { Date: 08/18/18 Time: } 10: 00 \\
\text { Sample: } 19712016 \\
\text { Included observations: } 43\end{array}$} \\
\hline \multicolumn{7}{|c|}{ Null hypothesis: No serial correlation at lag $\mathrm{h}$} \\
\hline Lag & LRE* stat $^{*}$ & df & Prob. & Rao F-stat & df & Prob. \\
\hline 1 & 1.206825 & 4 & 0.8770 & 0.299949 & $(4,68.0)$ & 0.8770 \\
\hline 2 & 1.979997 & 4 & 0.7394 & 0.494892 & $(4,68.0)$ & 0.7395 \\
\hline 3 & 0.909624 & 4 & 0.9232 & 0.225594 & $(4,68.0)$ & 0.9232 \\
\hline 4 & 2.104887 & 4 & 0.7165 & 0.526586 & $(4,68.0)$ & 0.7165 \\
\hline 5 & 1.888889 & 4 & 0.7562 & 0.471807 & $(4,68.0)$ & 0.7562 \\
\hline 6 & 1.901927 & 4 & 0.7538 & 0.475109 & $(4,68.0)$ & 0.7538 \\
\hline 7 & 1.119347 & 4 & 0.8912 & 0.278030 & $(4,68.0)$ & 0.8912 \\
\hline 8 & 4.526751 & 4 & 0.3394 & 1.152684 & $(4,68.0)$ & 0.3395 \\
\hline 9 & 7.146350 & 4 & 0.1284 & 1.855104 & $(4,68.0)$ & 0.1284 \\
\hline 10 & 1.707871 & 4 & 0.7893 & 0.426031 & $(4,68.0)$ & 0.7893 \\
\hline 11 & 4.540073 & 4 & 0.3378 & 1.156189 & $(4,68.0)$ & 0.3379 \\
\hline 12 & 6.983792 & 4 & 0.1367 & 1.810735 & $(4,68.0)$ & 0.1368 \\
\hline 13 & 1.875394 & 4 & 0.7587 & 0.468390 & $(4,68.0)$ & 0.7587 \\
\hline 14 & 0.087498 & 4 & 0.9991 & 0.021571 & $(4,68.0)$ & 0.9991 \\
\hline 15 & 2.147644 & 4 & 0.7086 & 0.537450 & $(4,68.0)$ & 0.7087 \\
\hline \multicolumn{7}{|c|}{ Null hypothesis: No serial correlation at lags 1 to $\mathrm{h}$} \\
\hline Lag & LRE $^{*}$ stat & df & Prob. & Rao F-stat & df & Prob. \\
\hline 1 & 1.206825 & 4 & 0.8770 & 0.299949 & $(4,68.0)$ & 0.8770 \\
\hline 2 & 4.134264 & 8 & 0.8448 & 0.509192 & $(8,64.0)$ & 0.8451 \\
\hline 3 & 4.499907 & 12 & 0.9726 & 0.358410 & $(12,60.0)$ & 0.9728 \\
\hline 4 & 4.875048 & 16 & 0.9963 & 0.281590 & $(16,56.0)$ & 0.9964 \\
\hline 5 & 8.818593 & 20 & 0.9849 & 0.404402 & $(20,52.0)$ & 0.9855 \\
\hline 6 & 12.89361 & 24 & 0.9678 & 0.488507 & $(24,48.0)$ & 0.9699 \\
\hline 7 & 18.49515 & 28 & 0.9130 & 0.602338 & $(28,44.0)$ & 0.9212 \\
\hline 8 & 18.97890 & 32 & 0.9668 & 0.515113 & $(32,40.0)$ & 0.9720 \\
\hline 9 & 23.88277 & 36 & 0.9393 & 0.569282 & $(36,36.0)$ & 0.9523 \\
\hline 10 & 29.15591 & 40 & 0.8974 & 0.616999 & $(40,32.0)$ & 0.9262 \\
\hline 11 & 36.20225 & 44 & 0.7921 & 0.695842 & $(44,28.0)$ & 0.8622 \\
\hline 12 & 46.46140 & 48 & 0.5360 & 0.843655 & $(48,24.0)$ & 0.6988 \\
\hline 13 & 50.65179 & 52 & 0.5270 & 0.800789 & $(52,20.0)$ & 0.7445 \\
\hline 14 & 58.04640 & 56 & 0.3998 & 0.816315 & $(56,16.0)$ & 0.7210 \\
\hline 15 & 63.79160 & 60 & 0.3447 & 0.747863 & $(60,12.0)$ & 0.7776 \\
\hline
\end{tabular}

\section{Anexo 9.}

Roots of Characteristic Polynomial Endogenous variables:

CRECIMIENTO_DEL_PIB_PER_CAP

ITA ANUAL TASA DE_CRECIMI

ENTO DEL NUMERO DE MATRIC

ULAS_EN_EDUCACION_SUPERI

Exogenous variables:

Lag specification: 12

Date: 08/18/18 Time: 10:12

\begin{tabular}{ll}
\hline \hline Root & Modulus \\
\hline \hline 1.000000 & 1.000000 \\
$0.499941-0.534689 \mathrm{i}$ & 0.732006 \\
$0.499941+0.534689 \mathrm{i}$ & 0.732006 \\
-0.681492 & 0.681492 \\
$-0.332271-0.585242 \mathrm{i}$ & 0.672988 \\
$-0.332271+0.585242 \mathrm{i}$ & 0.672988 \\
\hline \hline
\end{tabular}

VEC specification imposes 1 unit root(s).

\section{Anexo 10.}


VEC Residual Normality Tests

Orthogonalization: Cholesky (Lutkepohl)

Null Hypothesis: Residuals are multivariate normal

Date: 08/18/18 Time: 11:04

Sample: 19712016

Included observations: 43

\begin{tabular}{|c|c|c|c|c|}
\hline Component & Skewness & Chi-sq & df & Prob. ${ }^{*}$ \\
\hline 1 & -0.688779 & 3.399986 & 1 & 0.0652 \\
\hline 2 & 0.514049 & 1.893764 & 1 & 0.1688 \\
\hline Joint & & 5.293750 & 2 & 0.0709 \\
\hline Component & Kurtosis & Chi-sq & df & Prob. \\
\hline 1 & 3.824111 & 1.216827 & 1 & 0.2700 \\
\hline 2 & 2.853868 & 0.038260 & 1 & 0.8449 \\
\hline Joint & & 1.255087 & 2 & 0.5339 \\
\hline
\end{tabular}

\begin{tabular}{cccc} 
Component & Jarque-Bera & df & Prob. \\
\hline \hline 1 & 4.616813 & 2 & 0.0994 \\
2 & 1.932024 & 2 & 0.3806 \\
\hline \hline Joint & 6.548838 & 4 & 0.1617 \\
\hline
\end{tabular}

*Approximate p-values do not account for coefficient estimation

\section{Anexo 11.}

VEC Residual Heteroskedasticity Tests (Levels and Squares)

Date: 08/18/18 Time: 11:05

Sample: 19712016

Included observations: 43

Joint test:

\begin{tabular}{ccc}
\hline \hline Chi-sq & df & Prob. \\
\hline \hline 28.32551 & 30 & 0.5532 \\
\hline \hline
\end{tabular}

Individual components:

\begin{tabular}{llllll}
\hline \hline Dependent & R-squared & $F(10,32)$ & Prob. & Chi-sq(10) & Prob. \\
\hline \hline res1*res1 & 0.422708 & 2.343125 & 0.0331 & 18.17646 & 0.0521 \\
res2*res2 & 0.131385 & 0.484027 & 0.8879 & 5.649564 & 0.8438 \\
res2*res1 & 0.313558 & 1.461717 & 0.1993 & 13.48298 & 0.1979 \\
\hline \hline
\end{tabular}


VEC Residual Heteroskedasticity Tests (Includes Cross Terms)

Date: 08/18/18 Time: 11:07

Sample: 19712016

Included observations: 43

Joint test:

\begin{tabular}{ccc}
\hline \hline Chi-sq & df & Prob. \\
\hline \hline 66.39221 & 60 & 0.2662 \\
\hline \hline
\end{tabular}

Individual components:

\begin{tabular}{lccccc}
\hline \hline Dependent & R-squared & $F(20,22)$ & Prob. & Chi-sq(20) & Prob. \\
\hline \hline res1*res1 & 0.723109 & 2.872679 & 0.0091 & 31.09368 & 0.0540 \\
res2*res2 & 0.488358 & 1.049939 & 0.4533 & 20.99938 & 0.3972 \\
res2*res1 & 0.688439 & 2.430607 & 0.0229 & 29.60287 & 0.0765 \\
\hline \hline
\end{tabular}

